\title{
INPP5K and Atlastin-1 maintain the nonuniform distribution of ER-plasma membrane contacts in neurons
}

\author{
Jingbo Sun ${ }^{1, \star} \mathbb{\infty}$, Raihanah Harion ${ }^{1, \star}$, Tomoki Naito $^{1} \mathbb{B}$, Yasunori Saheki ${ }^{1,2}(\mathbb{0}$
}

\begin{abstract}
In neurons, the ER extends throughout all cellular processes, forming multiple contacts with the plasma membrane (PM) to fine-tune neuronal physiology. However, the mechanisms that regulate the distribution of neuronal ER-PM contacts are not known. Here, we used the Caenorhabditis elegans DA9 motor neuron as our model system and found that neuronal ER-PM contacts are enriched in soma and dendrite and mostly absent in axons. Using forward genetic screen, we identified that the inositol 5-phosphatase, CIL-1 (human INPP5K), and the dynamin-like GTPase, ATLN-1 (human Atlastin-1), help to maintain the non-uniform, somatodendritic enrichment of neuronal ER-PM contacts. Mechanistically, CIL-1 acts upstream of ATLN-1 to maintain the balance between ER tubules and sheets. In mutants of CIL-1 or ATLN-1, ER sheets expand and invade into the axon. This is accompanied by the ectopic formation of axonal ERPM contacts and defects in axon regeneration following laserinduced axotomy. As INPP5K and Atlastin-1 have been linked to neurological disorders, the unique distribution of neuronal ER-PM contacts maintained by these proteins may support neuronal resilience during the onset and progression of these diseases.
\end{abstract}

DOI 10.26508/lsa.202101092 | Received 12 April 2021 | Revised 3 September 2021 | Accepted 11 September 2021 | Published online 23 September 2021

\section{Introduction}

The ER is the largest membranous organelle, comprising a single continuous network of interconnected tubules and cisternae that contain multiple domains with different functions (Shibata et al, 2009; Westrate et al, 2015). Extending from the nuclear envelope, the peripheral ER consists of flat cisternae sheets and reticulated tubules that include two functionally segregated domains: rough and smooth ER (Shibata et al, 2006). Rough ER, characterized by ribosome-rich areas, is a major site for the synthesis of secreted and integral membrane proteins and is found primarily around the nucleus as stacked sheets connected by twisted membrane surfaces (Terasaki et al, 2013). By contrast, ribosome-free smooth ER is a major site for the synthesis of membrane lipids and for $\mathrm{Ca}^{2+}$ storage (Schwarz \& Blower, 2016). In addition, the ER extends throughout the cell, interacting with all other cellular organelles and membranes without membrane fusion via membrane contact sites to control cell physiology, including $\mathrm{Ca}^{2+}$ homeostasis, organelle dynamics, lipid exchange, and cell signaling (Phillips \& Voeltz, 2016; Valm et al, 2017; Wu et al, 2018).

In neurons, a continuous network of ER tubules and cisternae can be found throughout the neuronal soma, axon, and dendrites (Tsukita \& Ishikawa, 1976; Terasaki et al, 1994; Wu et al, 2017; Farias et al, 2019). Whereas soma and dendrites contain both rough and smooth ER, axonal ER comprises a network of largely smooth tubular ER with a very small diameter $(\sim 30 \mathrm{~nm}$ ) (ER tubules typically have a diameter of $\sim 60 \mathrm{~nm}$ in most cell types) (Wu et al, 2017; Yalcin et al, 2017; Terasaki, 2018). At the cell periphery, the ER is in close contact (within 10-30 nm) with the plasma membrane (PM) (Orci et al, 2009; Friedman \& Voeltz, 2011; West et al, 2011; FernandezBusnadiego et al, 2015; Bayer et al, 2017; Wu et al, 2017; Collado et al, 2019; Hoffmann et al, 2019). Growing evidence suggests that ER-PM contacts contribute to general cell physiology, as well as to the unique functional properties of neurons, including the control of neuronal excitability via local regulation of ion channel function and the facilitation of non-vesicular lipid transport between the ER and PM (Moriguchi et al, 2006; Kakizawa et al, 2007; de Juan-Sanz et al, 2017; Saheki \& De Camilli, 2017a; Johnson et al, 2018; Chen et al, 2019; Kirmiz et al, 2019; Sun et al, 2019; Vierra et al, 2019; Stefan, 2020). Thus, neuronal ER-PM contacts likely play important roles in neuronal survival and functional homeostasis. However, the molecular mechanisms by which the distribution of neuronal ER-PM contacts is maintained are currently unknown.

ER-PM contacts are mediated by various tethering proteins, including the evolutionarily conserved ER-resident proteins, the extended-synaptotagmins (E-Syts). In mammals, these are E-Syt1, E-Syt2, and E-Syt3; they are called tricalbins in yeast (Manford et al, 2012; Toulmay \& Prinz, 2012; Saheki, 2017; Saheki \& De Camilli, 2017a, 2017b; Collado et al, 2019; Hoffmann et al, 2019). E-Syts are anchored to ER membranes through their $\mathrm{N}$-terminal hydrophobic stretch and form homo- and hetero-meric complexes. They tether the ER to the PM via interactions between their cytosolic $\mathrm{C} 2$ domains and phosphatidylinositol 4,5-bisphosphate $\left[\mathrm{PI}(4,5) \mathrm{P}_{2}\right]$ within the $\mathrm{PM}$

${ }^{1}$ Lee Kong Chian School of Medicine, Nanyang Technological University, Singapore ${ }^{2}$ Department of Molecular Physiology, Faculty of Life Sciences, Kumamoto University, Kumamoto, Japan 
(Giordano et al, 2013; Fernandez-Busnadiego et al, 2015). E-Syts additionally possess a lipid-harboring synaptotagmin-like mitochondriallipid-binding (SMP) domain and mediate transport/exchange of glycerolipids between the ER and PM (Schauder et al, 2014; Saheki et al, 2016; Jeyasimman \& Saheki, 2019).

In contrast, the unique structure of the ER is maintained by a number of different proteins that function to control ER shape (i.e., ER-shaping proteins). Each ER-shaping protein participates in unique steps during the formation and maintenance of the peripheral ER network, including maintenance of ER tubules via curvature stabilization, connection of ER tubules via facilitation of homotypic ER membrane fusion, and maintenance of three-way junctions that result from the fusion of ER tubules (Shibata et al, 2009; Westrate et al, 2015). Many of ER-shaping proteins are evolutionarily conserved, and studies from various model organisms, including yeast, mammalian cells, flies, and worms, have demonstrated that functional dysregulation of ER-shaping proteins, or imbalances in their activities, results in abnormal ER structures, and in many cases, alters the abundance of cortical ER (i.e., the ER that mediates ER-PM contacts). In yeast, depletion of Reticulons/RTNs and/or REEPS/DP1/Yop1p family members, which are evolutionarily conserved ER-shaping proteins involved in curvature stabilization/generation of ER tubules, leads to loss of ER tubules, expansion of ER sheets, and an increase in ER-PM contacts (De Craene et al, 2006; Voeltz et al, 2006; Hu et al, 2008; West et al, 2011). In Drosophila neurons, these proteins are required for maintaining the tubular structure of the ER, including axonal ER (O'Sullivan et al, 2012; Yalcin et al, 2017; Espadas et al, 2019). On the other hand, depletion of Atlastins/ATLs, which are dynamin-like GTPases that facilitate homotypic fusion of ER tubules, results in unbranched ER tubules, loss of three-way junctions, and fragmentation of the ER in mammalian cells, flies, and worms (Hu et al, 2009; Orso et al, 2009; Summerville et al, 2016; Wang et al, 2016; Liu et al, 2019). In yeast, depletion of Sey1p (the dynamin-like GTPase that resembles the Atlastin) has no effect, but simultaneous depletion of Sey1p with either Yop1p or Rtn1p results in loss of ER tubules and expansion of cortical ER sheets, suggesting the potential importance of Atlastin family proteins in regulating ER tubule formation as well as cortical ER abundance (Hu et al, 2009; Anwar et al, 2012). A recent study demonstrated that INPP5K, a metazoan specific inositol 5-phosphatase that can be targeted to the $E R$, is required for the maintenance of $E R$ tubules in HeLa cells as well as in Caenorhabditis elegans PVD neurons (Dong et al, 2018). In the absence of INPP5K, ER sheets dramatically expand, although the precise mechanism of this process is still unclear (Dong et al, 2018). Notably, mutations in humans that impair the phosphatase activity of INPP5K lead to congenital muscular dystrophy with additional clinical manifestations, including intellectual impairments, suggesting that INPP5K plays an important role in the nervous system to maintain proper function of neurons (Osborn et al, 2017; Wiessner et al, 2017). Mutations in Atlastin-1, RTN2, or REEP1, also result in neurological problems; deleterious mutations in these proteins lead to hereditary spastic paraplegia, which is characterized by progressive loss of axons associated with motor neurons (Blackstone, 2012). Thus, proteins that are involved in the maintenance of proper ER shape are important for neuronal function and survival in humans. However, the relationship between these ERshaping proteins and the distribution and function of neuronal ER-PM contacts has remained elusive.
In this study, we explored the molecular mechanisms controlling the distribution of neuronal ER-PM contacts and found that ERshaping proteins play a critical role in this process. Using the $C$. elegans cholinergic DA9 motor neuron as a model system, we visualized the distribution of neuronal ER-PM contacts in vivo by fluorescence microscopy. In DA9, ER-PM contacts contain ESYT-2, the sole E-Syt homolog in C. elegans, and localize predominantly to somatodendritic regions, with very few ER-PM contacts present in the dorsal axon. We performed an unbiased forward genetic screen to identify mutants that mislocalized ER-PM contacts to the dorsal axon, and isolated mutations in at $n-1$ and cil-1, which encode homologs of mammalian Atlastin-1 and INPP5K, respectively. Our genetic and cell biological analysis revealed that CIL-1 acts upstream of ATLN-1 to maintain the balance between tubules and sheets at the cortical ER as well as to restrict the ER sheets to somatodendrites. In the absence of these proteins, cortical ER sheets expand and rough ER proteins localize ectopically to axonal ER. Supporting the importance of maintaining the balance between ER tubules and sheets, mutants that lack RET-1 (the sole reticulon homolog in C. elegans), whose depletion is known to result in ER sheet expansion, phenocopy the ectopic formation of ER-PM contacts observed in at $n-1$ and cil-1 mutants. Furthermore, we performed laser axotomy experiments in C. elegans, and found that regeneration of the DA9 axon was significantly reduced in both cil-1 and at $n-1$ mutants compared with wild type. These results suggest that the non-uniform distribution of neuronal ER-PM contacts that is maintained by CIL-1 and ATLN-1 is critical for the function of these contacts, potentially contributing to the resilience of neurons against neuronal damage.

\section{Results}

\section{ER-PM contacts are non-uniformly distributed in the $C$. elegans DA9 neuron}

To visualize neuronal ER-PM contacts in live neurons in vivo, we chose $C$. elegans as a model system because of its highly stereotypic cell lineage, well-defined neuroanatomy, and transparent nature. We focused our analysis on the DA9 cholinergic motor neuron, which localizes to the tail of the worm. DA9 has a simple cytoarchitecture. Its cell body resides in the ventral side of the tail; a dendrite extends anteriorly along the ventral nerve cord; and an axon extends into the dorsal nerve cord forming "en passant" presynaptic nerve terminals (Fig 1A) (Klassen \& Shen, 2007; Saheki \& Bargmann, 2009).

To label ER-PM contacts, a split GFP approach was used (Kamiyama et al, 2016; Romei \& Boxer, 2019) (Fig 1B and C). Previous studies have demonstrated that membrane contact sites can be labelled and visualized using this approach (Cieri et al, 2018; Kakimoto et al, 2018; Shai et al, 2018). We further extended this system to label ER-PM contacts in C. elegans neurons. We generated transgenic worms that co-expressed two components of split GFP under the DA9-selective itr-1pB promoter and performed imaging analysis using spinning disc confocal (SDC) microscopy or SDC structural illumination microscopy (SDC-SIM). One component of 

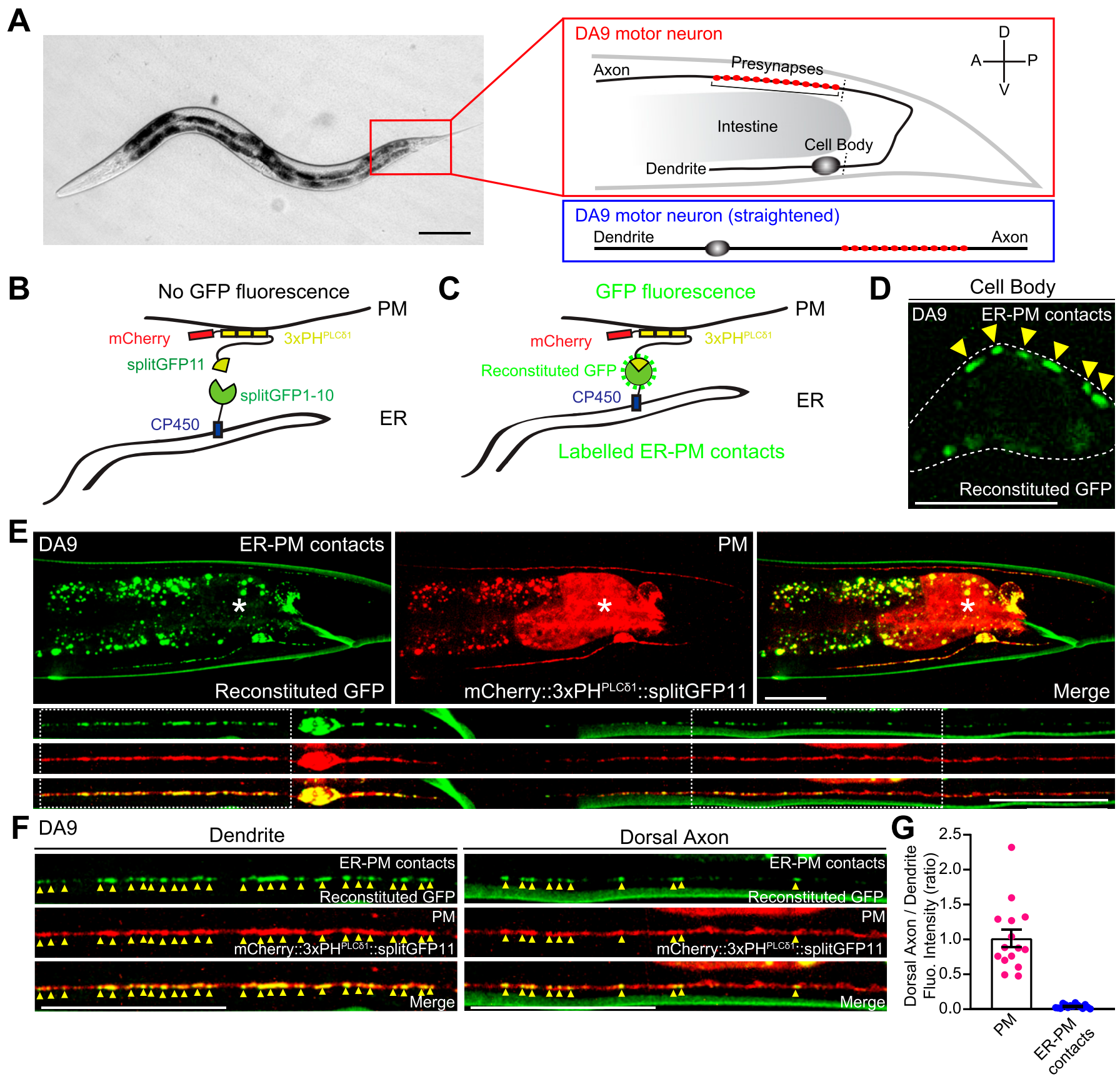

Figure 1. ER-PM contacts are non-uniformly distributed in the C. elegans DA9 neuron.

(A) Left: A hermaphrodite worm at L4 stage. Right: A schematic of the anatomy of DA9 motor neuron, located at the tail (top), and a schematic of straightened DA9 (bottom). Presynaptic nerve terminals present in the dorsal axon are indicated by red circles. Scale bar, $100 \mu \mathrm{m}$. (B, C) Schematics of the split GFP strategy for labelling the contacts between the ER and the plasma membrane (PM) in vivo. One component of split GFP (splitGFP11) was tagged to mCherry-fused with tandem pleckstrin homology (PH) domains of phospholipase C (PLC) $\delta 1$ (mCherry:3XPH ${ }^{\text {PLC } 1 . .: S p l i t G F P 11) ~ t h a t ~ b i n d ~ t o ~ P I(4,5) P ~}$ on the PM, and the other split GFP component (splitGFP1-10) was tagged to the cytosolic region of ER resident protein CP450 (CP450::splitGFP1-10). Close proximity of the two split GFP components results in GFP reconstitution, labelling ER-PM contact sites as green puncta. (D) A representative live spinning disc confocal structural illumination microscopy (SDC-SIM) image of the cell body of a DA9 neuron,

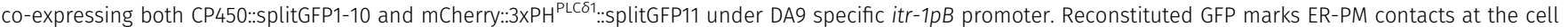
periphery (yellow arrowheads). A single focal plane of the center of the cell body is shown. White dashed line outlines the shape of the DA9 cell body. Scale bar, $5 \mu \mathrm{m}$.

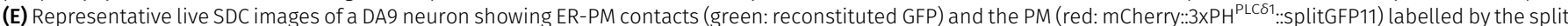
GFP approach. Bottom rows show straightened images of the same DA9 neuron. Dendrite and dorsal axon, as indicated by white dashed boxes, are enlarged in (F). The central autofluorescent region is the intestine (asterisk). Scale bars, $20 \mu \mathrm{m}$. (F) ER-PM contacts that are marked by the split GFP approach (yellow arrowheads) are nonuniformly distributed in DA9. Note their enrichment in dendrite compared to dorsal axon. Scale bars, $20 \mu \mathrm{m}$. (G) Quantification of the ratio of fluorescence intensity of

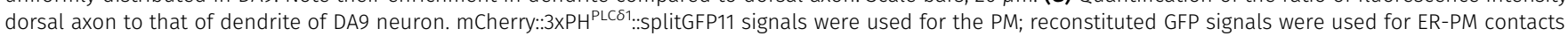
(mean $\pm \mathrm{SEM}, \mathrm{n}=15$ for both PM and ER-PM contacts).

Source data are available for this figure. 
the split GFP (fragments 1-10 of GFP) was fused to the cytosolic region of the ER membrane protein, CP450. The other GFP fragment (fragment 11) was fused to an mCherry-tagged PM binding module [3 $\times$ pleckstrin homology $(\mathrm{PH})$ domains derived from phospholipase $C(\mathrm{PLC})$ 81, which bind to $\mathrm{PM} \mathrm{PI}(4,5) \mathrm{P}_{2}$ ] (mCherry::3xPH ${ }^{\mathrm{PLC} \delta 1 .:}$ splitGFP11) (Fig 1B and C). Close apposition of these two fragments resulted in the assembly of fluorescent GFP as distinct puncta (observed by SDC-SIM) at the periphery of the DA9 cell body (Fig 1D). Thus, we were able to successfully label neuronal ER-PM contacts. Notably, reconstituted GFP signals labelling ER-PM contacts showed a non-uniform distribution; they were more abundant in the soma, dendrite, and proximal/ventral axon (all within the ventral region of the animal) compared with the circumferential axon and distal/dorsal axon. In contrast, mCherry-tagged $\mathrm{PH}^{\mathrm{PLC} \delta 1}$ signal, corresponding to the $\mathrm{PM}$, was present throughout the DA9 neuron (Fig 1E-G). Replacing mCherry::3xPH ${ }^{P L C \delta 1 .: . S p l i t G F P 11 ~ w i t h ~ c y t o s o l i c ~ m C h e r r y-t a g g e d ~ s p l i t ~ G F P ~}$ (fragment 11) (mCherry::splitGFP11) resulted in uniform distribution of GFP fluorescence throughout all neuronal processes of the DA9 neuron, demonstrating that CP450 is distributed throughout neuronal processes as a general ER marker (thus ruling out the biased enrichment of CP450 in the dendrite or ventral axon) (Fig S1A-D). These results suggest that ER-PM contacts are non-uniformly distributed in the $C$. elegans DA9 neuron.

\section{ESYT-2 localizes to neuronal ER-PM contacts}

The split GFP approach mostly labels ER-PM contacts that depend on the presence of $\mathrm{PM} \mathrm{PI}(4,5) \mathrm{P}_{2}$ [as mCherry::3xPH ${ }^{\mathrm{PLC} \delta 1}$ :.:splitGFP11 recognizes $\left.\mathrm{PM} \mathrm{PI}(4,5) \mathrm{P}_{2}\right]$. In addition, this approach may also force the formation of ectopic ER-PM contacts that may not reflect endogenous distribution of these contacts. ER-PM contacts are mediated by a number of ER-resident tethering proteins, including the evolutionarily conserved family of E-Syt proteins (Saheki \& De Camilli, 2017a, 2017b). In C. elegans, ESYT-2 is the sole ortholog of the E-Syt proteins and is structurally similar to mammalian E-Syt2 and E-Syt3, which localize constitutively to ER-PM contacts (Giordano et al, 2013). However, the subcellular distribution of ESYT-2 in neurons has not yet been studied. To further confirm the non-uniform distribution of ER-PM contacts that we observed with the split GFP approach in DA9 neuron, we visualized the endogenous localization of ESYT-2 using a cell-type specific endogenous labelling approach (Schwartz \& Jorgensen, 2016). In this approach, "FLP-on GFP" was fused to the N terminus of ESYT-2 by CRISPR/ Cas9-based gene editing. We visualized endogenous ESYT-2 (endoGFP::ESYT-2) specifically in the DA9 neuron by expressing FLP via the DA9-selective itr-1pB promoter. Puncta of endoGFP:: ESYT-2 were found primarily in the soma, dendrite, and ventral axon of DA9 (Fig 2A and B). Quantification of the fluorescence intensity showed that endoGFP::ESYT-2 puncta were much more abundant in the dendrite compared to the dorsal axon, similar to the distribution of ER-PM contacts observed using the split GFP approach (Fig 2A-C, compare with Fig 1E-G). A few endoGFP.::ESYT-2 puncta were also present in the dorsal axon. To determine if they localized to presynaptic regions of the DA9 axon, a synaptic vesicle marker [mCherry fused to RAB-3 (mCherry::RAB-3)] was co-expressed in DA9. Co-localization of endoGFP::ESYT-2 and mCherry::RAB-3 was assessed by line scan analysis. This revealed that endoGFP::ESYT-2 puncta in the dorsal axon localized outside of the presynaptic regions labelled by mCherry::RAB-3 (Fig 2B). The endogenous localization of ESYT-2 in DA9 was further examined at different developmental stages (larval L2, L3, and L4 stages) (Fig S2A-C). We did not detect endoGFP.:ESYT-2 at the larval L2 stage, most likely due to very low expression of ESYT-2 at this stage of development (Fig S2A). At the larval L3 stage, endoGFP::ESYT-2 puncta were present in the ventral DA9 process (Fig S2B), and the number of the puncta increased as animals grew to L4 larva (Fig S2C). Few endoGFP::ESYT-2 puncta were detected in the dorsal axon regardless of the development stages of the animal. Thus, the non-uniform distribution of endoGFP::ESYT-2 in the DA9 neuron is established at the larval L3 stage and maintained through development to $L 4$ and adult stages. In the AWC olfactory sensory neurons, which reside in the head region, endoGFP.:ESYT-2 also localized predominantly to the dendrite, with very little signal within presynaptic regions, indicating that the non-uniform distribution of ESYT-2 is a general feature of C. elegans neurons (Fig S2D and E).

To gain further insights into the localization of ESYT-2, endoGFP:: ESYT-2 puncta within the dendrite and axon were individually examined by tracking their movement along the processes via kymograph analysis. ESYT-2 puncta in the dendrite were immobile over the 2-min imaging period. By contrast, ESYT-2 puncta in the dorsal axon were highly mobile and dynamic. After 2 min, most of the ESYT-2 puncta in the dorsal axon had moved away from their time 0 position (Fig 2D). These results suggest that dendritic ESYT-2 localizes to cortical ER and stably associates with ER-PM contacts, whereas the mobile ESYT-2 possibly indicates minor fractions of ESYT-2 that may participate in membrane contact sites formed between the ER and other organelles. To examine whether dendritic ESYT-2 localizes to ER-PM contacts, wrmScarlet tagged ESYT-2 (wrmScarlet::ESYT-2) and split GFP components (to label ER-PM contacts) were co-expressed in the DA9 neuron and their association was assessed by line-scan analysis. Although wrmScarlet:: ESYT-2 did not co-localize perfectly with GFP puncta (i.e., split GFPlabelled ER-PM contact sites), the majority of wrmScarlet::ESYT-2 co-localized with GFP puncta, confirming that dendritic ESYT-2 localizes primarily to ER-PM contacts (Fig 2E and F). These results are consistent with the heterogeneity of the ER-PM contacts as shown in recent studies (Saheki \& De Camilli, 2017a; Besprozvannaya et al, 2018).

Collectively, these results demonstrate that ER-PM contacts in DA9 neurons are highly enriched in the somatodendritic region and that these contacts contain an evolutionarily conserved ER-PM tethering protein, ESYT-2.

\section{ESYT-2 distribution is altered in cil-1 mutants}

To investigate the underlying molecular mechanism regulating the non-uniform distribution of neuronal ER-PM contacts, we performed an unbiased, forward genetic screen using a transgenic worm that expressed mNeonGreen-tagged ESYT-2 (mNeonGreen:: ESYT-2) together with a synaptic vesicle marker (mCherry::RAB-3) under the control of the DA9-selective itr-1pB promoter. This strain enhanced our ability to detect ESYT-2 to efficiently visualize ER-PM contacts in the DA9 neuron. mNeonGreen::ESYT-2 localized predominantly to the somatodendritic region (zone A) and the ventral 
A

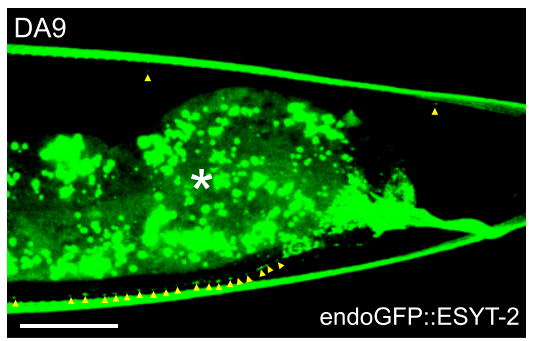

B
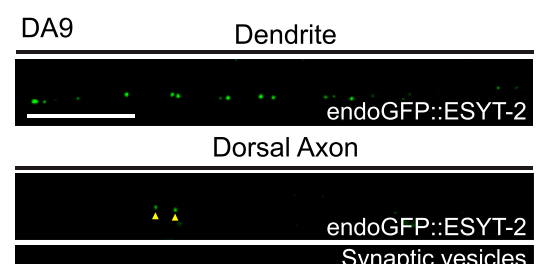

Synaptic vesicles
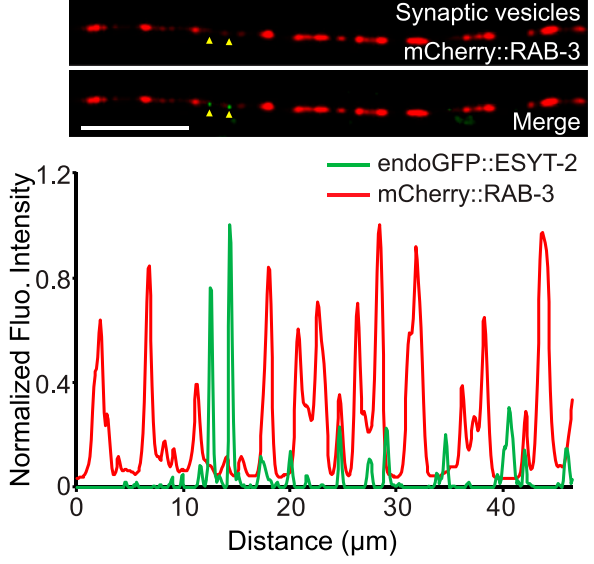

C endoGFP::ESYT-2 $\mathbf{D}$

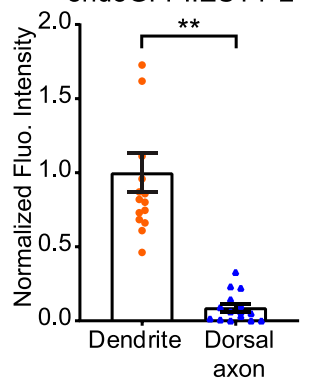

$E$
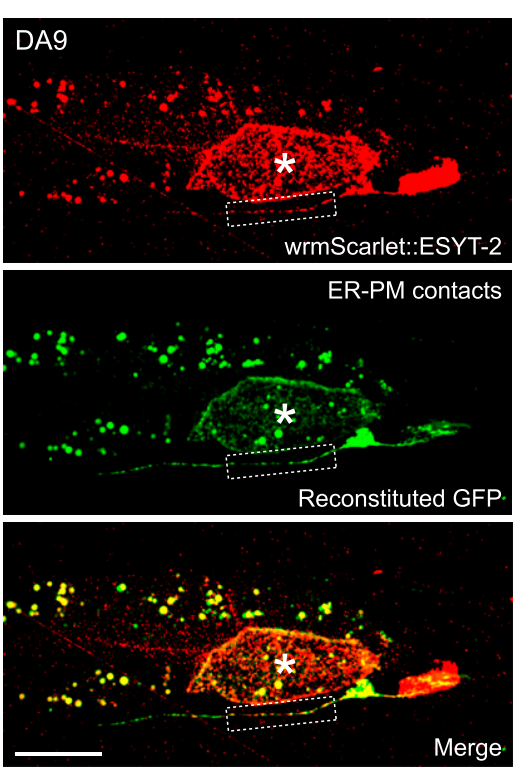

\section{Kymograph}

DA9 Dendrite

endoGFP::ESYT-2

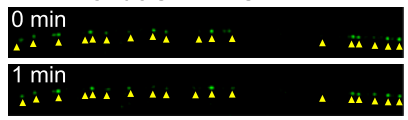

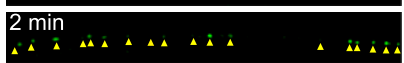

Kymograph

$\Delta$ inis

\section{.}

F DA9
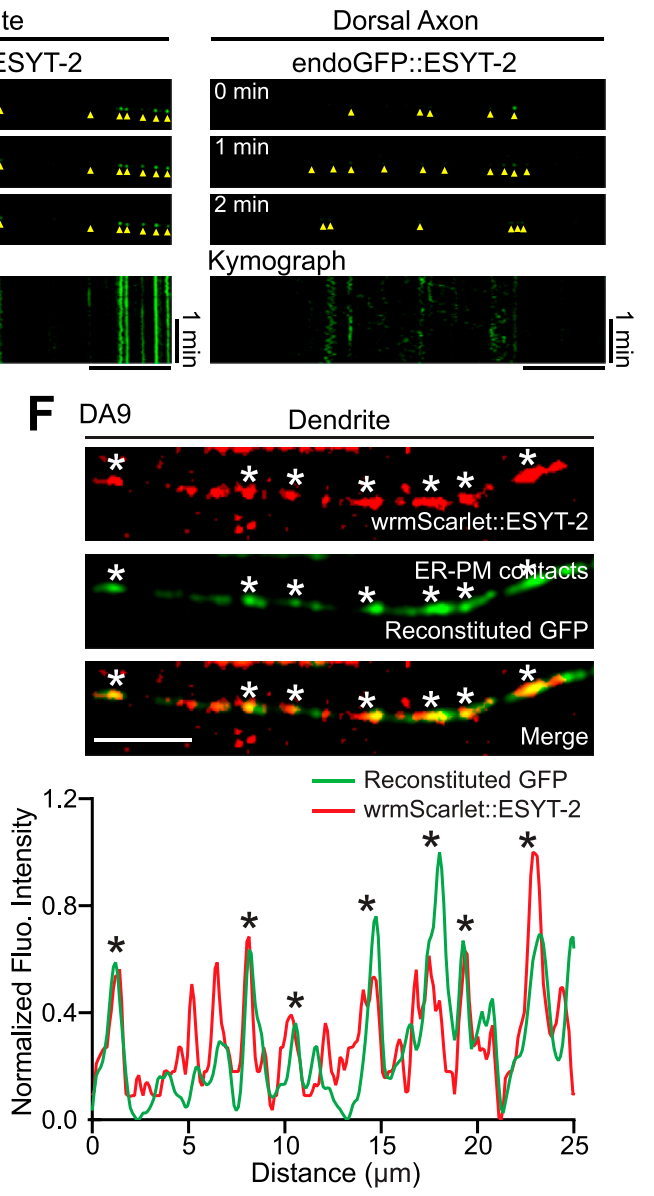

Figure 2. ESYT-2 localizes to neuronal ER-PM contacts.

(A) A representative live spinning disc confocal (SDC) image of a DA9 neuron, carrying endogenously tagged ESYT-2 (endoGFP::ESYT-2), showing enrichment of ESYT-2 in dendrite compared to dorsal axon. Yellow arrowheads indicate endoGFP.:ESYT-2 puncta. The central autofluorescent region is the intestine (asterisk). Scale bar, $20 \mu \mathrm{m}$. (B) Representative live SDC images of dendrite and dorsal axon of a DA9 neuron, co-expressing endoGFP::ESYT-2 and synaptic vesicle marker [mCherry-tagged with RAB-3 (mCherry::RAB-3)]. Yellow arrowheads indicate endoGFP::ESYT-2 puncta in dorsal axon. The line scan analysis of fluorescent intensity of endoGFP::ESYT-2 (green) and mCherry::RAB-3 (red) along the dorsal axon is shown below. Scale bars, $10 \mu \mathrm{m}$. (C) Quantification of normalized fluorescence intensity of endoGFP::ESYT-2 signals in dendrites and dorsal axons of DA9 neurons (mean $\pm \mathrm{SEM} ; \mathrm{n}=14$ for dendrite and $n=15$ for dorsal axon. Two-tailed unpaired $t$ test ** $P<0.0001$ ). (D) Kymographs showing the dynamics of endoGFP::ESYT-2 in dendrite (left) and dorsal axon (right) of a DA9 neuron. Images captured at different time points (0, 1, or 2 min) are shown as representative images. Yellow arrowheads indicate endoGFP.:ESYT-2 puncta. Note the disappearance of some endoGFP::ESYT-2 puncta in dorsal axon during the 2 min imaging period. Scale bars, $10 \mu \mathrm{m}$ and $1 \mathrm{~min}$. (E) Representative live SDC images of a DA9 neuron, co-expressing wrmScarlet-tagged ESYT-2 (wrmScarlet::ESYT-2) and the components for labelling ER-PM contacts (3XPH PLC81.:.SplitGFP11 and CP450::splitGFP1-10). Note the extensive overlap of wrmScarlet::ESYT-2 and reconstituted GFP (ERPM contacts). Dendrite of the DA9 neuron, as indicated by a white dashed box, is enlarged in (F). The central autofluorescent region is the intestine (asterisk). Scale bars, $20 \mu \mathrm{m}$. (F) Extensive overlap of wrmScarlet::ESYT-2 and reconstituted GFP (ER-PM contacts) in dendrite. The line scan analysis of normalized fluorescent intensity of reconstituted GFP (green) and wrmScarlet::ESYT-2 (red) along the dendrite is shown below. White and black asterisks mark the overlap. Scale bar, $5 \mu$ m.

Source data are available for this figure.

axon, but was generally excluded from the circumferential axon (zone $\mathrm{B}$ ) and the dorsal axon (zone $\mathrm{C}$ ), similar to the distribution of endoEGFP::ESYT-2 (Fig 3A).

From $\sim 1,000$ mutated haploid genomes, we isolated two mutants, yas37 and yas38, that exhibited increased levels of mNeonGreen::ESYT2 in the circumferential and dorsal axon (zone B and zone C), but normal RAB-3 puncta. yas37 was sterile, whereas yas38 produced a reduced brood size compared with controls (Fig S3A-E). Using balancer mapping and whole genome sequencing, we identified that yas37 carries a causative mutation in the cil-1 gene, which is the ortholog of mammalian INPP5K, and yas38 carries a causative mutation in the atln-1 gene, which is the ortholog of mammalian Atlastin-1.
In cil-1(yas37) mutants, mNeonGreen::ESYT-2 was uniformly distributed throughout the entire axon (Fig 3B). Furthermore, the dendritic ER, which was assessed by either mNeonGreen::ESYT-2 (Fig 3B and G) or a general ER marker CP450 (Fig S5A), was shortened compared with wild-type control. The fluorescence intensity of mNeonGreen::ESYT-2 was measured for each zone, and the ratio of fluorescence intensity in zone $\mathrm{C}$ to that in zone A was compared between control animals and cil-1(yas37) mutants (Fig 3C-F). In control animals, the ratio was $\sim 0.1$, indicating $\sim 10$-fold enrichment of mNeonGreen::ESYT-2 in the dendrite compared to the dorsal axon covered by presynaptic regions. This ratio was significantly increased in cil-1(yas37) mutants $(\sim 1)$, indicating the increased presence of 

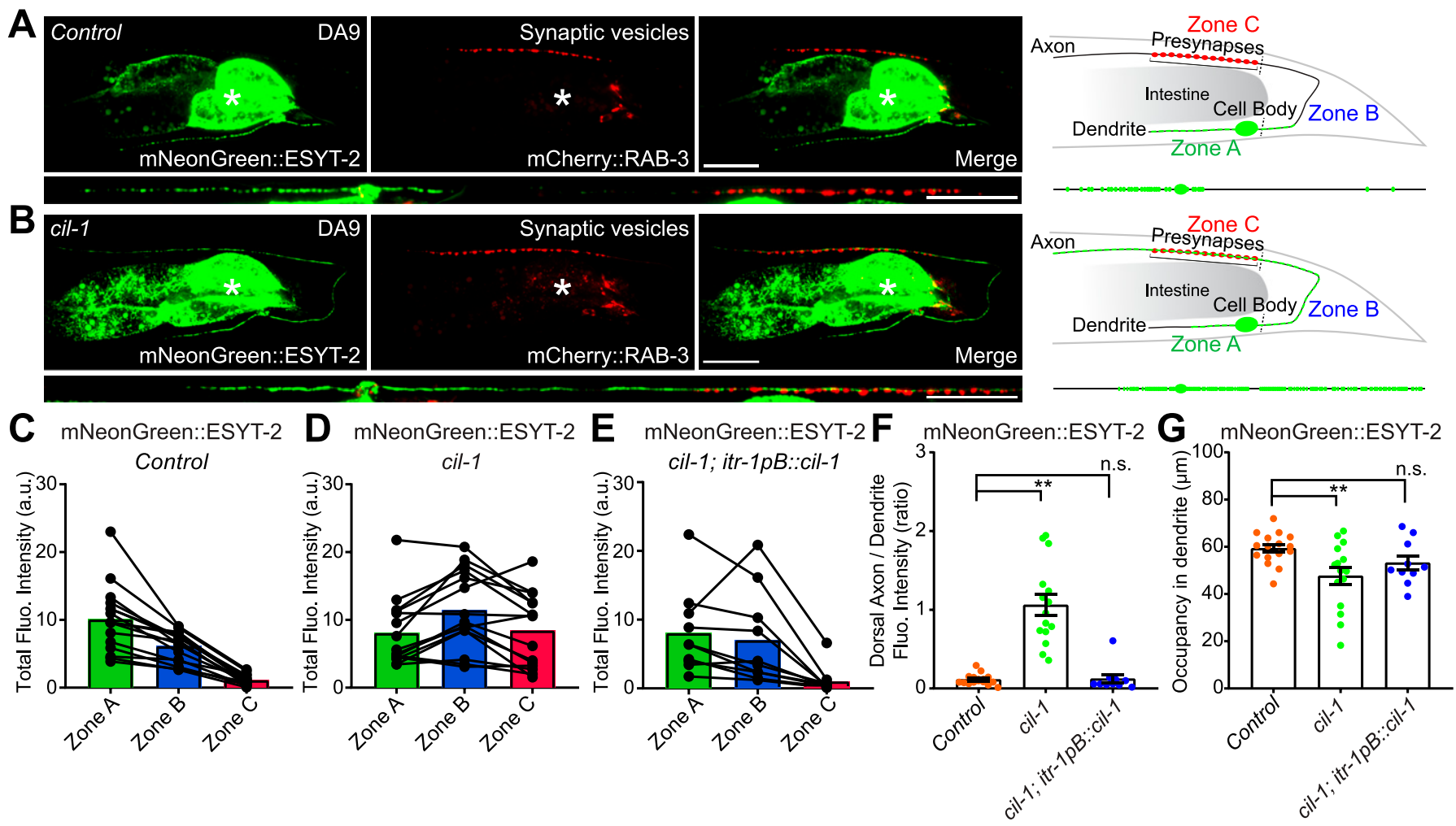

Figure 3. ESYT-2 distribution is altered in cil-1(yas37) mutants.

(A, B) Left: Representative live spinning disc confocal images of a DA9 neuron, co-expressing mNeonGreen-tagged ESYT-2 (mNeonGreen::ESYT-2) and mCherry::RAB-3 in wild-type control (A) and cil-1 (yas37) mutants (B). Straightened images of the DA9 neurons are shown below. Right: Schematics showing the distribution of mNeonGreen:: ESYT-2 (marked as small green circles) in DA9 neuron. Zone A denotes dendrite; Zone B denotes ventral axon and circumferential axon up to the most proximal region of presynaptic nerve terminals labelled by mCherry::RAB-3 (flanked by the two black dashed lines); Zone C denotes the region of dorsal axon that is covered by presynaptic nerve terminals. Note the altered distribution of mNeonGreen::ESYT-2 in cil-1 (yas37) mutants. The central autofluorescent region is the intestine (asterisk). Scale bars, $20 \mu \mathrm{m}$. (C, D, E) Quantification of total fluorescence intensity of mNeonGreen::ESYT-2 puncta present in zones A, B, and C in wild-type control ( $\mathrm{n}=16)(\mathrm{C})$, cil-1 (yas37) mutants $(n=15)(D)$, and cil-1 (yas37) mutants expressing CIL-1 under DA9 specific itr-1pB promoter $(n=10)(E)$. Each connected dot represents one animal. (F) Quantification of the ratio of mNeonGreen::ESYT-2 fluorescence intensity of dorsal axon (zone C) to that of dendrite (zone A) from the same animals as shown in $(C, D, E)$. (mean \pm SEM; Dunnett's multiple comparisons test ${ }^{* *} P<0.0001$ [Control versus cil-1 (yas37)], n.S. denotes not significant). (G) Quantification of the occupancy of mNeonGreen::ESYT-2 signals in dendrites (zone A) from the same animals as shown in (C, D, E). Note the rescue of the phenotype by DA9 specific re-expression of CIL-1 in cil-1(yas37) mutants in (E, F, G). (mean \pm SEM; Dunnett's multiple comparisons test; ${ }^{* *} P=0.0080$ [Control versus cil-1 (yas37)], n.S. denotes not significant). Source data are available for this figure.

mNeonGreen::ESYT-2 in the distal axon. Importantly, the mCherry:: RAB-3 signal was not affected in cil-1(yas37) mutants (Fig S3F), indicating that the mechanisms controlling the distribution of ESYT-2 is different from those that regulate the formation and/or assembly of presynaptic terminals. In addition, DA9-specific expression of cil-1 under the DA9-selective itr-1pB promoter restored the distribution of mNeonGreen::ESYT-2 (Fig 3E-G). These results suggest that cil-1 acted cell-autonomously in the DA9 neuron to maintain the distribution of ESYT-2.

\section{CIL-1 regulates the cortical ER network and maintains the distribution of ER-PM contacts in neurons}

cil-1 encodes an evolutionarily conserved inositol 5-phosphatase that acts primarily on phosphoinositide $\mathrm{PI}(4,5) \mathrm{P}_{2}$ (INPP5K in human). It contains a 5-phosphatase domain followed by a SKICH domain (Bae et al, 2009; Dong et al, 2018) (Fig 4A). Sequencing cil-1(yas37) DNA revealed a $\mathrm{G}$ to $A$ mutation, which results in an early stop codon in the
CIL-1 open reading frame. cil-1 mutants are recessive, suggesting that cil-1(yas37) is a loss-of-function allele of CIL-1 (Fig 4A).

To examine whether 5-phosphatase activity and/or the $\mathrm{SKICH}$ domain are necessary for $\mathrm{CIL}-1$ function, we tested mutant versions of CIL-1 proteins for their abilities to rescue mNeonGreen::ESYT-2 localization in DA9. A missense mutation in a critical residue of the 5-phosphatase domain (N175A) eliminated its activity, failing to rescue the mNeonGreen::ESYT-2 mislocalization in cil-1(yas37) mutant (Figs $4 \mathrm{~A}$ and $\mathrm{C}$ and S4A and C). Deletion of the SKICH domain also inactivated $\mathrm{CIL}-1$ (Figs $4 \mathrm{~A}$ and $\mathrm{C}$ and $\mathrm{S} 4 \mathrm{~A}$ and $\mathrm{C}$ ). These results suggest that both the 5-phosphatase activity and the SKICH domain are essential for CIL-1 function. Another cil-1 mutant, cil-1(my15), causes a premature stop in the $\mathrm{CIL}-1$ open reading frame, resulting in the deletion of the SKICH domain (Bae et al, 2009). cil-1(my15) mutants displayed uniform distribution of mNeonGreen::ESYT-2 along the dorsal axon and dendrite, similar to that seen in cil1(yas37) null mutants, highlighting the importance of the $\mathrm{SKICH}$ domain for CIL-1 function (Figs $4 \mathrm{~A}$ and $\mathrm{C}$ and S4B and C). 
A

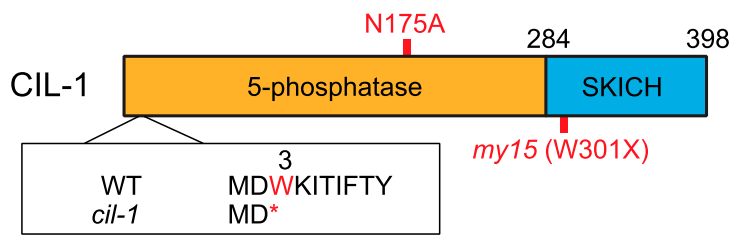

B
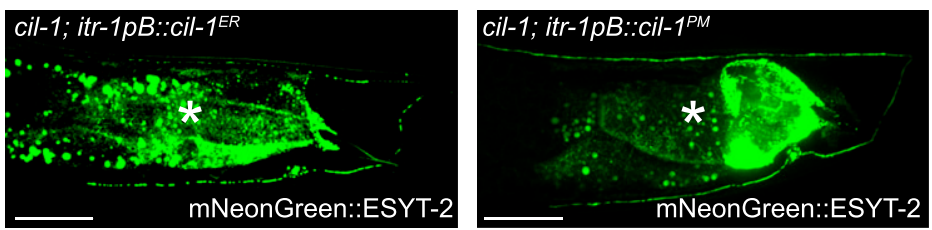

D

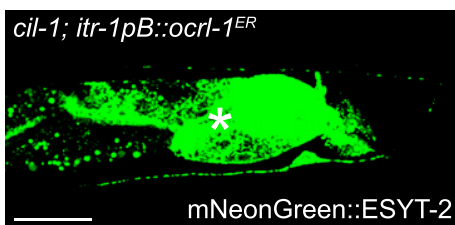

cil-1; itr-1pB::unc-26

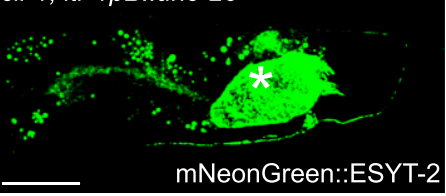

mNeonGreen::ESYT-2
E

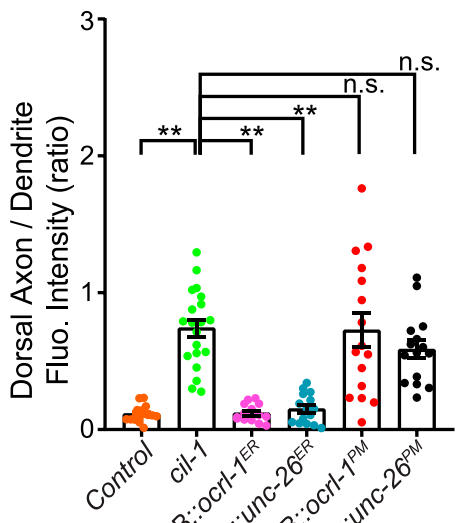

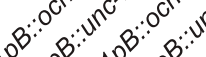

C

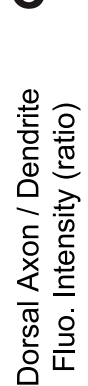
mNeonGreen::ESYT-2
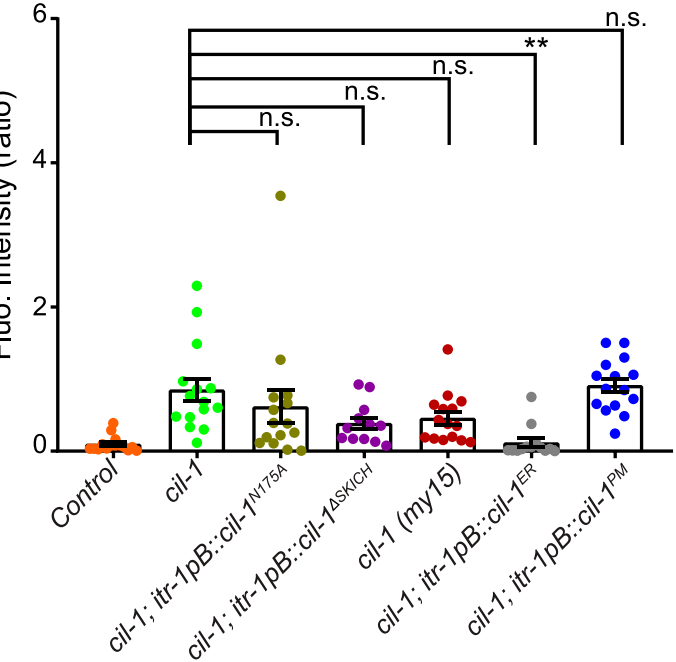

$\mathbf{F}$

mNeonGreen::ESYT-2 DA9 soma periphery

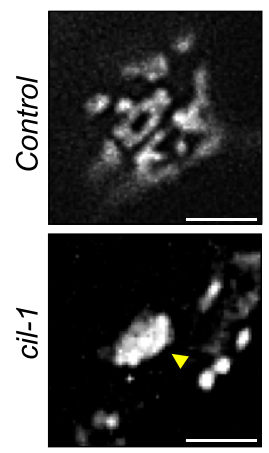

G mNeonGreen::ESYT-2

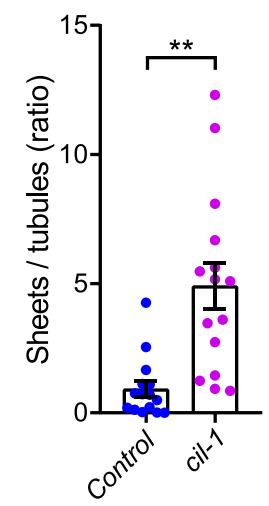

H

DA9

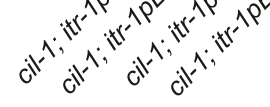

cil-1

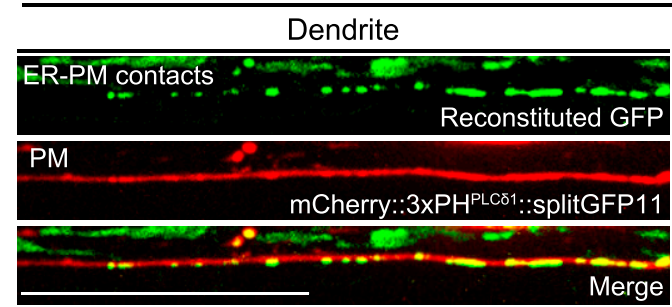

Dorsal Axon

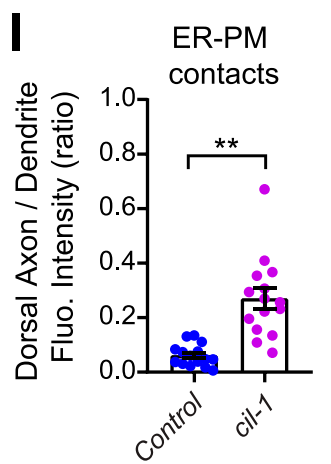

Figure 4. CIL-1 regulates the cortical ER network and maintains the distribution of ER-PM contacts in neurons.

(A) Domain structure of CIL-1 in C. elegans. N175A phosphatase-dead and early termination mutations (W3X [indicated by an asterisk], corresponding to cil-1 (yas37) mutation, and W301X, corresponding to my15) are indicated. (B) Representative live spinning disc confocal (SDC) images of DA9 neurons, expressing mNeonGreen::ESYT-2, from cil-1 (yas37) mutants that additionally express either ER-targeted CIL-1 (left) or PM-targeted CIL-1 (right) under DA9 specific itr-1pB promoter. Note that expression of ER-targeted CIL-1 restored the distribution of mNeonGreen::ESYT-2 in cil-1 (yas37) mutants. Scale bars, $20 \mu \mathrm{m}$. (C) Quantification of the ratio of mNeonGreen::ESYT-2 fluorescence intensity of dorsal axon (zone C) to that of dendrite (zone A) in wild-type control $(n=15)$, cil-1 (yas37) $(n=15)$, cil-1 (yas37); itr-1pB::cil- $1^{\text {N175A }}(n=15)$, cil-1 (yas37);

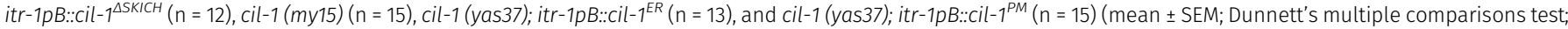

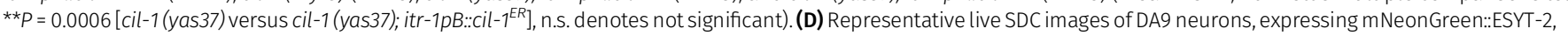
from cil-1 (yas37) mutants that additionally express ER-targeted phosphatase domains of either OCRL-1 or UNC-26 under DA9 specific itr-1pB promoter. Scale bars, $20 \mu \mathrm{m}$. (E) Quantification of the ratio of mNeonGreen::ESYT-2 fluorescence intensity of dorsal axon (zone C) to that of dendrite (zone A) in wild-type control $(n=15)$, cil- $1(y a s 37)(n=20)$,

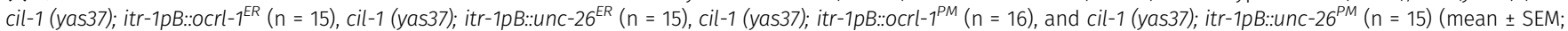
Dunnett's multiple comparisons test; ${ }^{* *} P<0.0001$ cil-1 (yas37) versus Control, cil-1 (yas37) versus cil-1 (yas37); itr-1pB:::0crl-1 ${ }^{E R}$, and cil-1 (yas37) versus cil-1 (yas37); 
The SKICH domain is required to recruit INPP5K/CIL-1 to various cellular compartments, including the ER, the PM, and the nucleus (Gurung et al, 2003; Dong et al, 2018). To determine the site at which CIL-1 functions to maintain the distribution of ESYT-2 in DA9, the 5-phosphatase domain of CIL-1 was selectively targeted to the ER or to the PM by fusing it with either the ER-resident CP450 protein or the PM-targeting motif of Lck. DA9-specific expression of the ERtargeted CIL-1 5-phosphatase domain restored the distribution of mNeonGreen::ESYT-2 in cil-1(yas37) mutants, whereas the PMtargeted CIL-1 5-phosphatase had no effect (Fig 4B and C). These results suggest that $\mathrm{CIL}-1$ may function at the ER to modulate $\mathrm{PI}(4,5)$ $\mathrm{P}_{2}$ levels of this organelle to maintain non-uniform distribution of ESYT-2 in neurons (see the Discussion section), although the possibility that $\mathrm{PM} \mathrm{PI}(4,5) \mathrm{P}_{2}$ plays some roles in regulating ESYT-2 distribution in neurons cannot be excluded.

To further investigate the role of $\mathrm{PI}(4,5) \mathrm{P}_{2}$ in the distribution of ESYT-2 in neurons, we targeted 5-phosphatase domains from other $\mathrm{PI}(4,5) \mathrm{P}_{2}$ phosphatases, namely, OCRL-1 and UNC-26 (orthologues of human OCRL and synaptojanin-1, respectively), to the ER or to the PM by the same strategy that we used for targeting CIL-15-phosphatase domain to these cellular compartments. HeLa cells were used to confirm the activities of these chimeric 5-phosphatase domains (Fig S4D-G). Expression of either PM-targeted OCRL-1 5-phosphatase domain (mScarlet-I-OCRL-1 ${ }^{\mathrm{PM}}$ ) or PM-targeted UNC26 5-phosphatase domain (mScarlet-I-UNC-26 ${ }^{\mathrm{PM}}$ ) strongly reduced the levels of $\mathrm{PM} \mathrm{PI}(4,5) \mathrm{P}_{2}$, as assessed by iRFP-tagged with the $\mathrm{PH}$

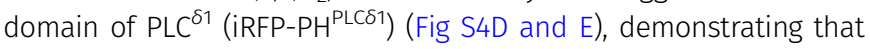
the 5-phosphatase domains of OCRL-1 and UNC-26 efficiently dephosphorylate $\mathrm{PI}(4,5) \mathrm{P}_{2}$ in cells. In contrast, expression of ER-targeted versions of the same 5 -phosphatase domains (OCRL- $1^{\mathrm{ER}}$-EGFP and UNC-26 ${ }^{\text {ER }}$-EGFP) had not effects on the levels of PM PI $(4,5) \mathrm{P}_{2}$ (Fig $\mathrm{S} 4 \mathrm{~F}$ and $\mathrm{G}$ ). These results suggest that the chimeric 5-phosphatase domains primarily act in cis without affecting $\mathrm{PI}(4,5) \mathrm{P}_{2}$ levels of other cellular compartments.

Finally, we expressed these chimeric 5-phosphatase domains specifically in DA9 neuron and examined whether forced reduction of $\mathrm{PI}(4,5) \mathrm{P}_{2}$ levels in the $\mathrm{PM}$ or ER membranes restore the nonuniform distribution of mNeonGreen::ESYT-2 in cil-1(yas37) mutants. Targeting the 5-phosphatase domain of either UNC-26 or OCRL-1 to the ER but not to the PM restored the distribution of mNeonGreen:: ESYT-2 to the similar levels to control animals (Fig 4D and E). These results suggest the importance of $\mathrm{PI}(4,5) \mathrm{P}_{2}$ levels in ER membranes for maintaining the non-uniform distribution of ESYT-2.

Notably, CIL-1/INPP5K function is critical for maintaining the balance between ER tubules and sheets (Dong et al, 2018). Depletion of INPP5K in HeLa cells leads to the reduction of ER tubules and expansion of ER sheets, whereas loss of CIL-1 activity in C. elegans disrupts the formation of ER tubules and impairs their extension into dendrites of the PVD neuron (Dong et al, 2018). However, the role of $\mathrm{CIL}-1 /$ INPP5K in maintaining the structure of cortical ER (i.e., the ER that is engaged in ER-PM contacts) remains unknown. To examine if $\mathrm{CIL}-1$ is required for regulating cortical ER structure in neurons, we imaged mNeonGreen::ESYT-2 under SDCSIM at the periphery of the DA9 soma as an estimate of the structure of cortical ER in DA9. In control animals, cortical ER, as assessed by mNeonGreen::ESYT-2, formed network of sheets and tubules (Fig 4F) (see the Materials and Methods section for quantification details). In cil-1(yas37) mutants, however, cortical ER was visibly less complex, with fewer tubules, consisting primarily of larger sheets that lacked fenestration (Fig $4 \mathrm{~F}$ and $\mathrm{G}$ ).

These results indicated that the abnormal expansion of cortical ER sheets in DA9 may have contributed to the ectopic localization of ESYT-2 to dorsal axons in cil-1(yas37) mutants. To confirm whether ER-PM contacts are indeed mislocalized to dorsal axons, we used the split GFP approach in DA9 neuron of cil-1(yas37) mutants. Remarkably, ER-PM contacts were abundant in the dorsal axon of DA9 in cil-1(yas37) mutants (Fig $4 \mathrm{H}$ and I). In addition, the reconstituted GFP signal was largely diffuse, indicating that the ectopically formed ER-PM contacts were mediated by expanded cortical ER sheets. Collectively, these results suggest that ER shape may play a role in maintaining the distribution of neuronal ER-PM contacts.

\section{CIL-1 functions upstream of ATLN-1 to maintain the distribution of ER-PM contacts in neurons}

The other mutant from the forward genetic screen, atln-1(yas38), showed defects in mNeonGreen::ESYT-2 distribution with normal mCherry::RAB-3 puncta that resembled those of cil-1(yas37) mutants (Figs 5A and B and S3F). In atln-1(yas38) mutants, mNeonGreen::ESYT2 was present uniformly throughout the entire axon (Fig 5A and B). In addition, the dendritic ER, as assessed by either mNeonGreen::ESYT-2 or a general ER marker CP450, was significantly shorter than in wildtype controls or cil-1(yas37) mutants (Figs 5A, E, and G and S5A). Expressing wild-type ATLN-1 via the DA9-selective itr-1pB promoter restored the mNeonGreen::ESYT-2 localization pattern in DA9, suggesting that atln-1 acts cell autonomously, as seen with cil-1 (Fig S5B-D, G, and H). ER-PM contacts, labelled by the split GFP approach, were also ectopically present in the dorsal axon in atln-1(yas38) mutants, further supporting that ATLN-1 and CIL-1 mediate similar functions (Fig 5C and D).

Sequencing the at In-1(yas38) mutant revealed a $\mathrm{G}$ to $\mathrm{A}$ mutation, which results in an amino acid substitution (E338K) in the ATLN-1 open reading frame (Fig S5B). at $n-1$ mutants are recessive, suggesting that at $n$-1(yas38) is a possible reduction-of-function allele of ATLN-1. at In-1 encodes a member of the evolutionarily conserved family of Atlastin proteins, which are ER-localized dynamin-like

itr-1pB::unc-26 $6^{E R}$, n.s. denotes not significant). (F) Representative live SDC-SIM images of the periphery of DA9 cell body, expressing mNeonGreen::ESYT-2, from wild-type control and cil-1 (yas37) mutants. Single focal planes are shown. A yellow arrowhead in a cil-1 (yas37) mutant soma denotes the accumulation of mNeonGreen::ESYT-2 in expanded sheet-like ER. Scale bars, $2 \mu \mathrm{m}$. (G) Quantification of the ratio of the total area of ER sheets to that of ER tubules, as assessed by mNeonGreen::ESYT-2 signals at the periphery of DA9 cell body, in wild-type control $(n=15)$ and cil-1 (yas37) $(n=15)$ (mean \pm SEM; Two-tailed unpaired $t$ test, $\left.{ }^{* *} P=0.0003\right)$. $(\mathbf{H})$ Representative live SDC images of a DA9 neuron, showing ER-PM contacts (green: reconstituted GFP) and the PM (red: mCherry::3xPH ${ }^{\text {PLC } 81 .: . S p l i t G F P 11) ~ t h a t ~ a r e ~ l a b e l l e d ~ b y ~ t h e ~ s p l i t ~ G F P ~ a p p r o a c h, ~ i n ~ d e n d r i t e ~ a n d ~}$ dorsal axon of cil-1 (yas37) mutants. Note the ectopic formation and expansion of ER-PM contacts in dorsal axon of cil-1 (yas37) mutants compared to wild-type control (compare with Fig 1E). Scale bars, $20 \mu \mathrm{m}$. (I) Quantification of the ratio of reconstituted GFP fluorescence intensity (ER-PM contacts) of dorsal axon to that of dendrite in DA9 neuron of wild-type control $(n=15)$ and cil-1 (yas37) $(n=15)$ (mean \pm SEM; Two-tailed unpaired $t$ test, $\left.{ }^{* *} P<0.0001\right)$.

Source data are available for this figure. 
A
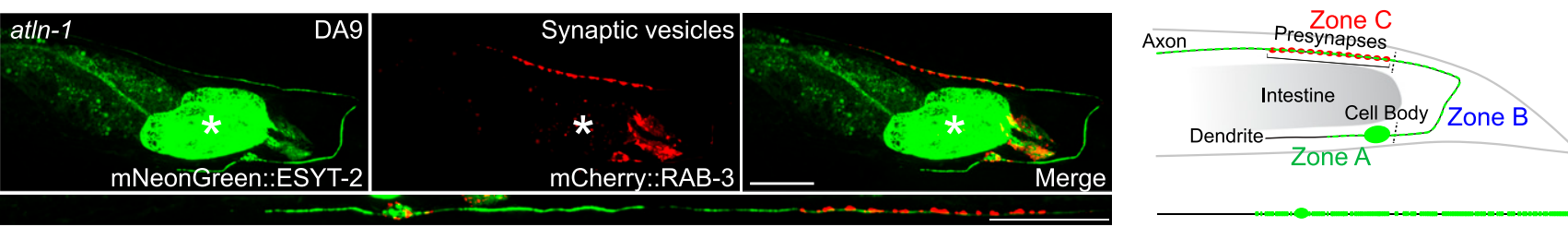

B
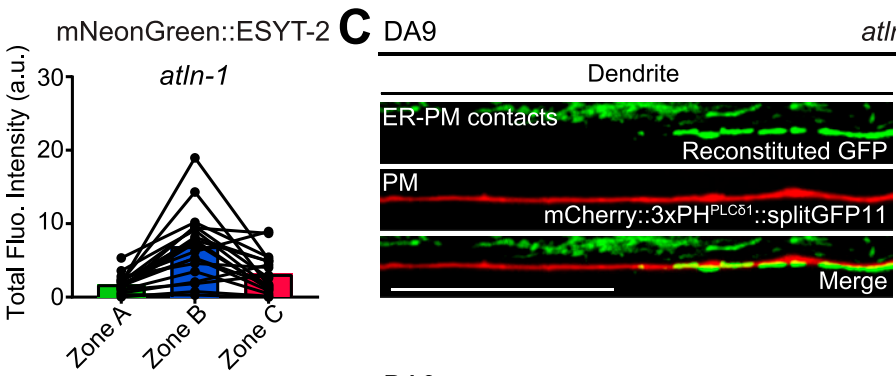

atln-1

D ER-PM contacts

$\mathbf{E}$ DA9
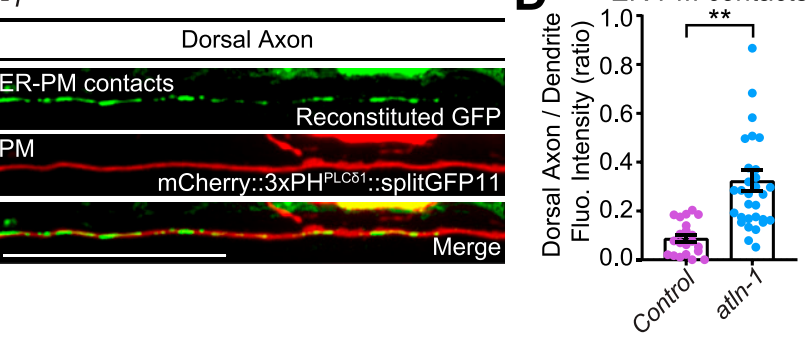

Control

cil-1

atln-1

cil-1; atln-1

cil-1; itr-1pB::atln-1 atIn-1; itr-1pB::cil-1

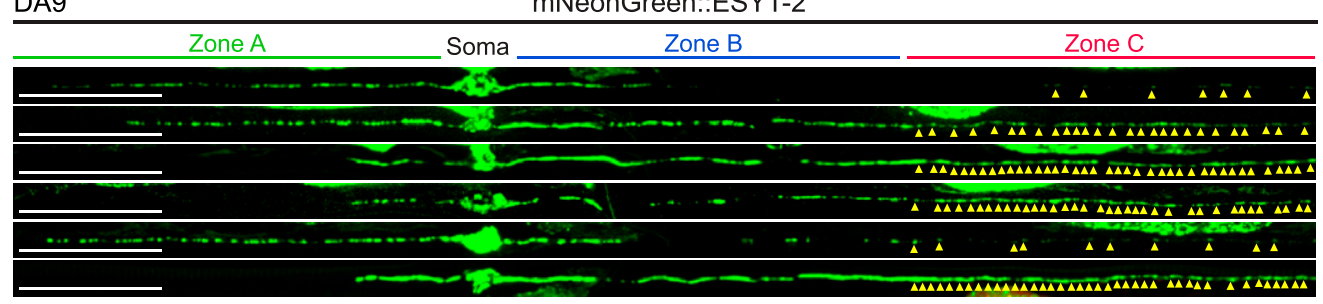

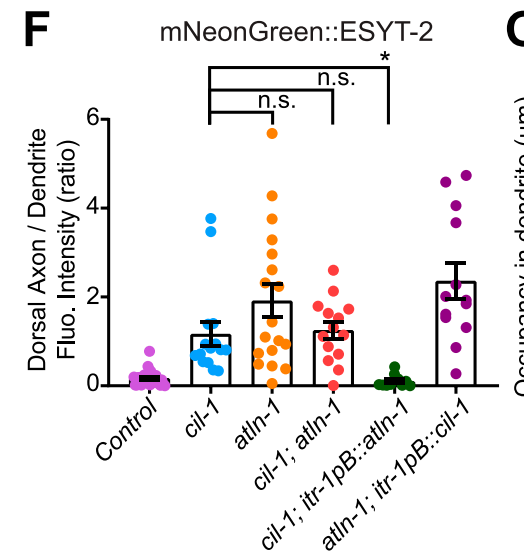

G mNeonGreen::ESYT-2

H

mNeonGreen::ESYT-2

I mNeonGreen::ESYT-2
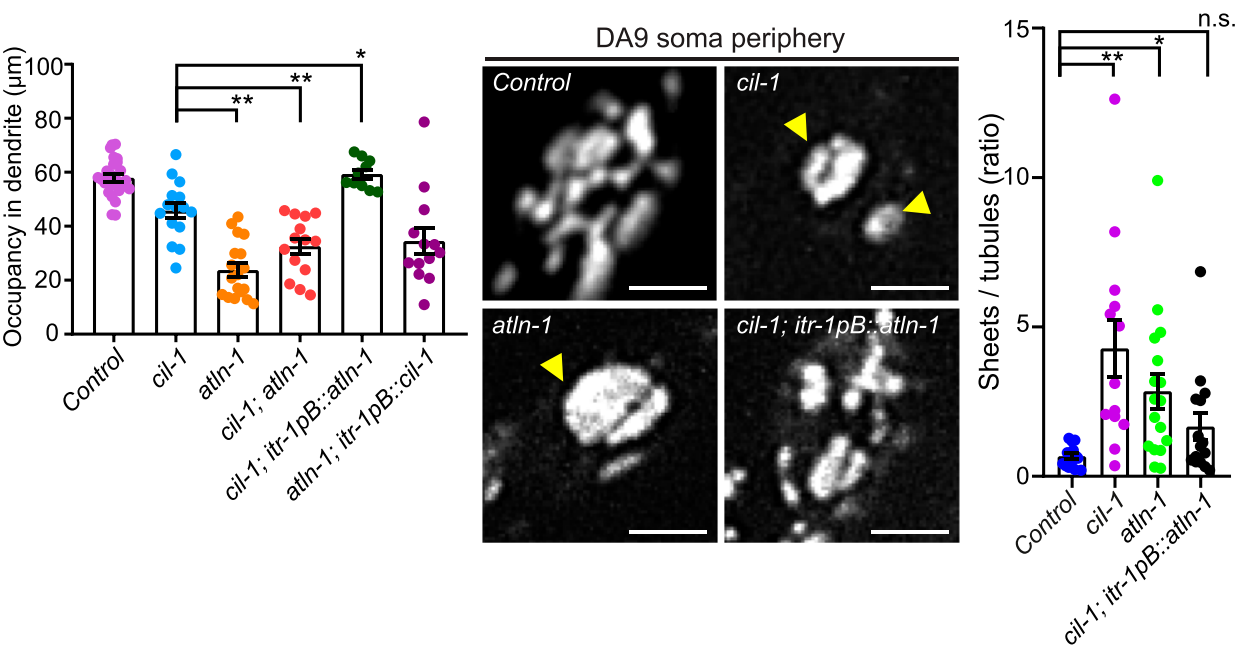

Figure 5. CIL-1 and ATLN-1 have related functions in maintaining ER shape in neurons.

(A) Left: Representative live spinning disc confocal (SDC) images of a DA9 neuron, co-expressing mNeonGreen::ESYT-2 and mCherry::RAB-3, in atln-1 (yas38) mutants. A straightened image of the DA9 neuron is shown below. Right: A schematic showing the distribution of mNeonGreen.:ESYT-2 (marked as small green circles) in DA9 neuron. Note the altered distribution of mNeonGreen::ESYT-2 in atln-1 (yas38) mutants compared to wild-type control (compare to Fig 3A). Scale bars, $20 \mu \mathrm{m}$. (B) Quantification of the total fluorescence intensity of mNeonGreen::ESYT-2 present in different zones of atln-1 (yas38) mutants $(n=18)$ shows increased abundance of ESYT-2 in zone $C$ compared to wild-type control (compare to Fig 3C). (C) Representative live SDC images of a DA9 neuron, showing ER-PM contacts (green: reconstituted GFP) and the PM

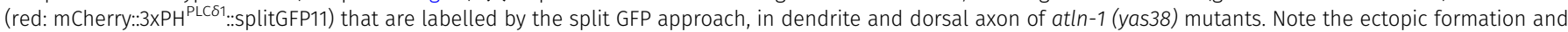
expansion of ER-PM contacts in dorsal axon compared to wild-type control (compare to Fig 1E). Scale bars, $20 \mu \mathrm{m}$. (D) Quantification of the ratio of reconstituted GFP fluorescence intensity (ER-PM contacts) of dorsal axon to that of dendrite in DA9 neuron of wild-type control $(n=21)$ and atln-1 (yas38) $(n=31)($ mean \pm SEM; Two-tailed unpaired $t$ test, $\left.{ }^{* *} P<0.0001\right)$. (E) Representative live SDC images of straightened DA9 neurons of animals with indicated genotype. Yellow arrowheads mark mNeonGreen: ESYT-2 signals in zone C. Note that overexpression of ATLN-1 in cil-1 (yas37) mutants suppressed the ectopic localization of mNeonGreen::ESYT-2 in zone C.

(F) Quantification of the ratio of mNeonGreen::ESYT-2 fluorescence intensity of dorsal axon (zone C) to that of dendrite (zone A) in DA9 neurons of animals as shown in (E): wlid-type control $(n=26)$, cil-1 (yas37) $(n=15)$, atln-1 (yas38) $(n=18)$, cil-1 (yas37); atln-1 (yas38) $(n=14)$, cil-1 (yas37); itr-1pB::atln-1 $(n=10)$, and atln-1 (yas38); itr-1pB::cil-1 $(n=13)$ (mean $\pm S E M$; Dunnett's multiple comparisons test; * $P=0.0432$ [cil-1 (yas37) versus cil-1 (yas37); itr-1pB::atln-1], n.s. denotes not significant). (G) Quantification of the occupancy of mNeonGreen::ESYT-2 signals in dendrites (zone A) of DA9 neurons from the same animals as in (F). (mean \pm SEM; Dunnett's multiple comparisons test; ${ }^{* *} P<0.0001$ [cil-1 (yas37) versus atln-1 (yas38)], ${ }^{* *} P=0.0054$ [cil-1 (yas37) versus cil-1 (yas37); atln-1 (yas38)], ${ }^{*} P=0.0108$ [cil-1 (yas37) versus cil-1 (yas37); itr-1pB::atln-1]). (H) Representative live SDC-SIM images of the periphery of DA9 cell body, expressing mNeonGreen::ESYT-2, from animals with indicated genotype. Single focal planes are shown. Yellow arrowheads in cil-1 (yas37) and atln-1 (yas38) mutants denote the accumulation of mNeonGreen::ESYT-2 in expanded sheet-like ER. Note the partial 
GTPases. Similar to other Atlastin proteins, ATLN-1 consists of a GTPase domain followed by a three-helix bundle (3HB), transmembrane (TM) segments, and a cytosolic tail (C-Tail) (Liu et al, 2019) (Fig $\mathrm{S5B}$ ). The glutamic acid that is mutated to lysine in the atln-1(yas38) mutant (E338K) is located near the 3HB domain, which is required for oligomerization of Atlastins (Bian et al, 2011; Byrnes \& Sondermann, 2011). Overexpression of the ATLN-1 (E388K) mutant protein partially restored the distribution of mNeonGreen::ESYT-2 in DA9 neurons, suggesting that ATLN-1 (E388K) retains some function (Fig S5B-D, G, and $H$ ). DA9-specific overexpression of an ATLN-1 variant (K80A) with dominant-negative GTPase activity (Liu et al, 2019) in wild-type animals resulted in altered distribution of mNeonGreen::ESYT-2, supporting the critical role of ATLN-1's GTPase activity in maintaining a normal distribution of ESYT-2 in DA9 neurons (Fig S5B and E-H).

To gain further insight into the relationship between cil-1 and at $n-1$, we performed genetic epistasis analysis between these mutants. cil-1(yas37); atln-1(yas38) double mutants displayed defects in the distribution of mNeonGreen::ESYT-2, similar to atln-1(yas38) single mutants (Fig $5 \mathrm{E}-\mathrm{G}$ ), consistent with related functions of cil-1 and atln-1. Strikingly, overexpression of atln-1 in DA9 of cil-1(yas37) mutants suppressed the ectopic accumulation of mNeonGreen:: ESYT-2 in the dorsal axon (zone C) and partially restored the distribution of mNeonGreen::ESYT-2 in the dendrite (zone A) (Fig 5E-G). In contrast, cil-1 overexpression failed to rescue mNeonGreen::ESYT-2 distribution in atln-1(yas38) mutants (Fig 5E-G). These results demonstrate that ATLN-1 acts genetically downstream of CIL-1.

Genetic interaction between cil-1 and at $n$-1 suggests that proteins encoded by these genes may function together to maintain the structure of cortical ER. Thus, we further examined the relationship between these proteins by imaging cortical ER. We used SDC-SIM to visualize mNeonGreen::ESYT-2 at the periphery of the DA9 cell body. Notably, at $n$-1(yas38) mutants displayed dramatic expansion of cortical ER sheets, similar to cil-1(yas37) mutants (Fig $5 \mathrm{H}$ and I). Overexpression of ATLN-1 in cil-1(yas37) mutants partially restored the balance between ER tubules and sheets and suppressed the expansion of cortical ER sheets. These results indicate that ATLN-1 overexpression, at least partially, bypasses the function of CIL-1 in maintaining the network of cortical ER tubules and sheets (Fig $5 \mathrm{H}$ and I). Collectively, our results demonstrate that ATLN-1 functions downstream of CIL-1 to maintain the structure of cortical ER, and further suggest that the expansion of cortical ER sheets, as observed in cil-1 and atln-1 mutants, underlies ectopic formation of ER-PM contacts in neuronal axons.

\section{Cortical ER shape is important for the normal distribution of ER-PM contacts in neurons}

Our results suggested that cortical ER shape is potentially important for maintaining the distribution of neuronal ER-PM contacts. To further test this idea, we analyzed mutants that lacked the curvature-stabilizing ER-shaping protein, reticulon. Yeast mutants that lack reticulons have fewer cortical ER tubules and more cortical ER sheets (De Craene et al, 2006; Voeltz et al, 2006; West et al, 2011). Consistent with these yeast studies, C. elegans mutants lacking reticulon, ret-1(tm390) (RET-1 is the sole homolog of reticulons in C. elegans), had fewer cortical ER tubules and enlarged cortical ER sheets (as assessed by mNeonGreen::ESYT-2) in the DA9 soma, similar to cil-1 or atln-1 mutants (Fig 6A). Furthermore, the dendritic ER, which was assessed by mNeonGreen::ESYT-2, was shortened compared with wildtype control similar to these mutants (Fig S6B). Importantly, ret-1(tm390) mutants also exhibited an altered distribution of mNeonGreen::ESYT-2 in DA9 neuron, where mNeonGreen::ESYT-2 was present uniformly throughout the entire axon (Figs 6B and C and S6A). ER-PM contacts, labelled by the split GFP approach, were also ectopically present in the dorsal axon in ret-1(tm390) mutants (Fig 6D and E).

To further confirm the role of cortical ER shape in the distribution of ER-PM contacts in neurons, we overexpressed CLIMP-63, a luminal ER spacer whose overexpression is known to result in expansion of ER sheets in mammalian cells (Shibata et al, 2010) (there is no homolog of CLIMP-63 in C. elegans), in DA9 neurons and examined its effect on the distribution of ESYT-2. CLIMP-63 overexpression resulted in expansion of cortical ER sheets and reduction in cortical ER tubules, as assessed by mNeonGreen::ESYT-2, in the DA9 soma, similar to cil-1, atln-1, and ret-1 mutants (Fig S6C). Notably, this was accompanied by altered distribution of mNeonGreen::ESYT-2 in DA9 axons; mNeonGreen::ESYT-2 became more abundant in the commissure region of DA9 (and less restricted to the somatodendritic region of DA9) compared to wildtype control (Fig S6D-F). These results suggest that the abundance of cortical ER sheets affects the distribution of ER-PM contacts in neurons. However, we could not detect the increased presence of mNeonGreen::ESYT-2 in the dorsal axons of DA9 in this condition (Fig S6G). This could be possibly due to preferential targeting of CLIMP-63 in somatodendritic regions of neurons as previously reported (Farias et al, 2019).

Taken together, these data support the notion that cortical ER shape, and more specifically the proper balance of ER tubules and sheets, play a role in restricting the distribution of neuronal ER-PM contacts to specific regions within neurons.

\section{Expansion and invasion of ER sheets in dorsal axon contribute to abnormal distribution of neuronal ER-PM contacts}

Using electron microscopy to visualize ER structure in C. elegans neurons poses a challenge due to its small size. In C. elegans neurons, the ribosome-rich domain of the ER, or rough ER, is found primarily in the soma and is generally absent from the axon (Rolls et al, 2002; Saheki \& Bargmann, 2009). Thus, we visualized the distribution of fluorescent protein-labelled rough ER proteins via fluorescent microscopy and examined whether ER sheets invaded into the dorsal axons of DA9 in cil-1, atln-1, and ret-1 mutants.

rescue of cortical ER structure in cil-1 (yas37) mutants overexpressing ATLN-1 under DA9 specific itr-1pB promoter. Scale bars, $2 \mu \mathrm{m}$. (I) Quantification of the ratio of the total area of ER sheets to that of ER tubules, as assessed by mNeonGreen::ESYT-2 signals at the periphery of DA9 cell body, in wild-type control $(n=14)$, cil-1 (yas37) $(n=13)$, atln-1 (yas38) $(n=17)$, and cil-1 (yas37); itr-1pB::atln-1 $(n=15)$ (mean \pm SEM; Dunnett's multiple comparisons test; ${ }^{* *} P=0.0004\left[\right.$ Control versus cil-1 (yas37)], ${ }^{\star} P=0.0265$ [Control versus atln-1 (yas38)], n.s. denotes not significant).

Source data are available for this figure. 
A

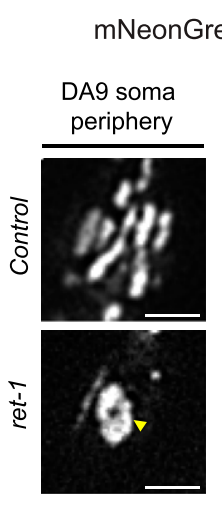

B

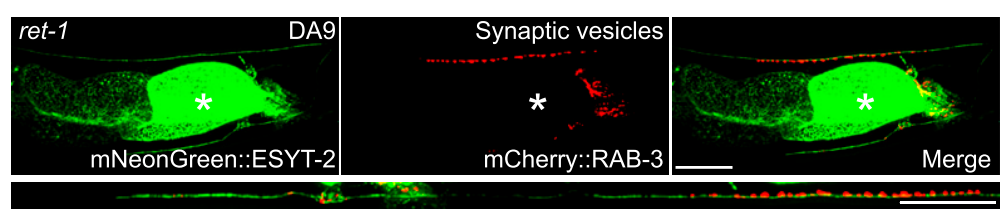

C mNeonGreen::ESYT-2

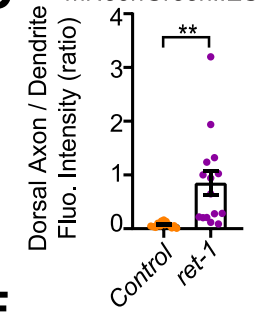

E

ret-1

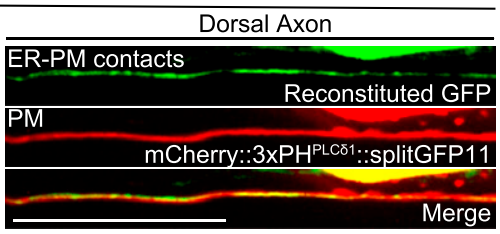

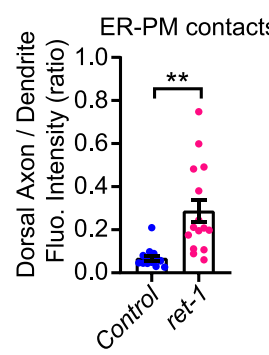

F DA9

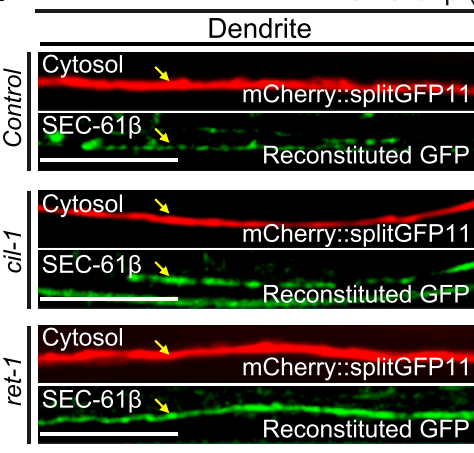

I

DA9

TRAP $\beta:: m N e o n G r e e n$ (rough ER)

\begin{tabular}{ll}
\hline \multicolumn{2}{c}{ Dendrite } \\
\hline Control _. \\
\hline cil-1 \\
\hline ret-1 \\
\hline
\end{tabular}

G

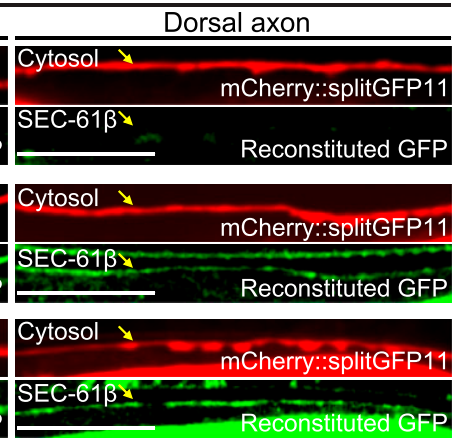

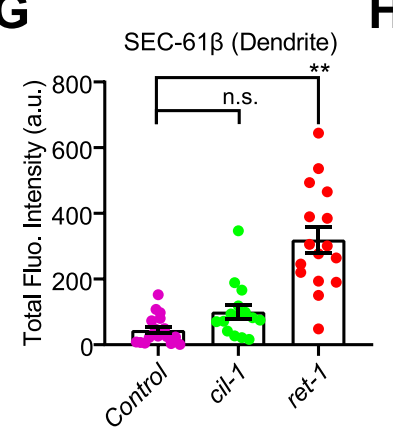

H
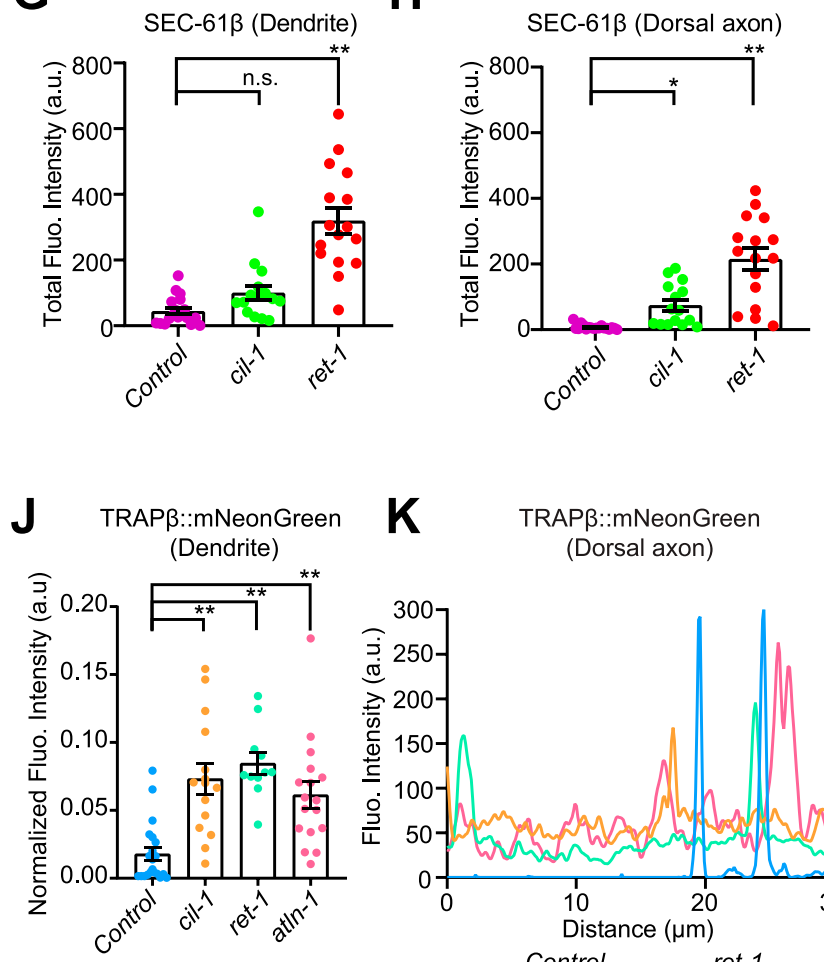

(Dorsal axon)

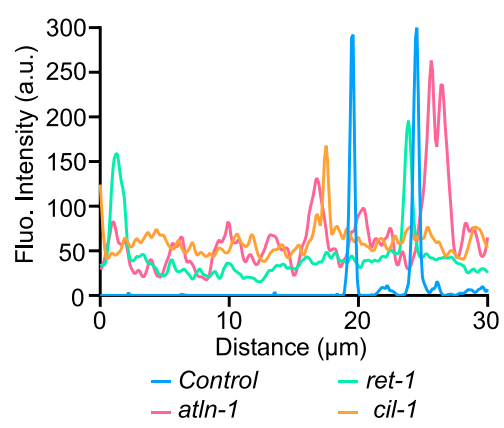

Figure 6. Abnormal distribution of neuronal ER-PM contacts is accompanied by expansion of ER sheets in dorsal axon.

(A) Left: Representative live spinning disc confocal (SDC)-SIM images of the periphery of DA9 cell body, expressing mNeonGreen::ESYT-2, in wild-type control and ret-1 (tm390) mutants. Single focal planes are shown. A yellow arrowhead denotes the accumulation of mNeonGreen::ESYT-2 in expanded sheet-like ER. Scale bars, $2 \mu \mathrm{m}$. Right: Quantification of the ratio of the total area of ER sheets to that of ER tubules, as assessed by mNeonGreen::ESYT-2 signals at cell periphery, in wild-type control $(n=15)$ and ret-1 $(t m 390)(n=16)\left(\right.$ mean $\pm S E M$; two-tailed unpaired $t$ test, $\left.{ }^{*} P=0.0146\right)$. (B) Representative live SDC images of a DA9 neuron, co-expressing mNeonGreen::ESYT-2 and mCherry::RAB-3, from ret-1 (tm390) mutants. A straightened image of the DA9 neuron is shown below. The central autofluorescent region is the intestine (asterisk). Scale bars, $20 \mu \mathrm{m}$. (C) Quantification of the ratio of mNeonGreen::ESYT-2 fluorescence intensity of dorsal axon (zone $\mathrm{C}$ ) to that of dendrite (zone A) of DA9 in wild-type control ( $\mathrm{n}=$ 15) and ret-1 $(\operatorname{tm} 390)(n=15)$ (mean $\pm S E M$; Two-tailed unpaired $t$ test, $\left.{ }^{* *} P=0.0015\right)$. (D) Representative live SDC images of dendrite and dorsal axon of DA9, showing ERPM contacts (green: reconstituted GFP) and the PM (red: mCherry:3XPH PLCS1::splitGFP11) labelled by the split GFP approach in ret-1 (tm390) mutants. (E) Quantification of the ratio of reconstituted GFP fluorescence intensity (ER-PM contacts) of dorsal axon to that of dendrite in DA9 of wild-type control $(n=15)$ and ret-1 $(t m 390)(n=15)$ (mean $\pm \mathrm{SEM}$; Two-tailed unpaired $t$ test, $\left.{ }^{*} P=0.0004\right)$. (F) Representative live SDC images of DA9 neurons, carrying endogenously tagged splitGFP1-10::SEC-61 $\beta$ and additionally expressing mCherry-tagged splitGFP11 (mCherry::splitGFP11) under DA9 selective mig-13 promoter, showing the endogenous localization of SEC-61 $\beta$ (green: reconstituted GFP) and the cytosol (red: mCherry::SplitGFP11) in animals of indicated genotype. Yellow arrows indicate the neuronal processes. Scale bars, $10 \mu \mathrm{m}$. (G) Quantification of the total fluorescence intensity of reconstituted GFP (endogenous SEC-61 $\beta$ ) in dendrite of wild-type control $(n=18)$, cil-1 (yas37) ( $n=15)$, and ret-1 $(t m 390)(n=16)$ (mean $\pm S E M$; Dunnett's multiple comparisons test; ${ }^{\star \star} P<0.0001$ [Control versus ret-1 (tm390)], n.s. denotes not significant). (H) Quantification of the total 
In the first set of experiments, we endogenously tagged the cytosolic $N$ terminus of rough ER marker SEC-61 $\beta$ (SEC61 translocon $\beta$ subunit) with one component of split GFP (fragments 1-10) using CRISPR-based gene-editing. We then expressed the other component of split GFP (fragment 11) together with soluble mCherry using the DA9-specific mig-13 promoter. Thus, we could visualize endogenously tagged SEC-61 $\beta$ via GFP reconstitution specifically in the mCherry-labelled DA9 neuron (Fig 6F-H). In wild-type controls, SEC-61 $\beta$ was mostly present in the soma, with little in the dendrite and none in the axon (Figs $6 \mathrm{~F}-\mathrm{H}$ and $\mathrm{S} 6 \mathrm{H}$ ). However, in ret1(tm390) and cil-1(yas37) mutants, SEC-61 $\beta$ was clearly present in the axon, and was also increased in the dendrite. In contrast, SEC$61 \beta$ levels were not affected in the soma (Fig S6H). As the sec-61.B gene is less than 1 centimorgan from the atln-1 gene, we were unable to establish a strain carrying the sec-61.B gene tagged with the split GFP component in the atln-1 mutant.

In the second set of experiments, we expressed a version of translocon-associated protein $\beta$ (TRAP $\beta$ ), another rough ER marker, in which its $C$-terminus was tagged with mNeonGreen, in DA9 via the DA9-specific itr-1pB promoter (Fig 6I-K). In control animals, TRAP $\beta$ :: mNeonGreen primarily localized to the cell body with little presence in the dendrite or axon. In the dorsal axon, TRAP $\beta$ ::mNeonGreen localized to a few distinct puncta, suggesting that it accumulates in specific compartments within axonal ER (Fig 6I-K). In cil-1(yas37), ret-1(tm390), and atln-1(yas38) mutants, however, TRAP $\beta:: m N e o n G r e e n$ was much more abundant in the dendrite and more diffusely localized within the axon compared with control animals. Levels of TRAP $\beta$ ::mNeonGreen in the soma were not affected (Fig S6I).

Collectively, these results show that ER sheets expanded and invaded into the dorsal axon and became more abundant in dendrite of DA9 neuron in cil-1(yas37), ret-1(tm390), and atln1(yas38) mutants. Our data strongly suggest that these mutants exhibited ectopically formed ER-PM contacts in the dorsal axon because of expansion and invasion of cortical ER sheets in this region.

\section{ER-PM contacts regulated by CIL-1 and ATLN-1 are important for efficient axon regeneration}

Axon regeneration is an important physiological response to various insults to neurons. The role of ER-PM contacts in this process remains unclear. After laser-induced damage to the DA9 dorsal axon, it has previously been shown that the axon proximal to the damage grows back (i.e., regenerates). In ric-7(n2657) mutants, the axon distal to the damage degenerates much more quickly than wild-type animals, eliminating potential interference from the remaining distal axon fragments after the damage (Ding \& Hammarlund, 2018) (Fig 7A-C).
Metazoan ER-PM contacts are populated by various tethering proteins, including E-Syts and Junctophilins. Interestingly, the absence of Junctophilin (JPH-1 in C. elegans), which is a metazoanspecific ER-PM tethering protein, negatively affected axon regeneration for the $C$. elegans mechanosensory PLM neuron, after laser-induced axonal injury. In contrast, esyt-2 is dispensable for axon regeneration of the PLM neuron (Kim et al, 2018). We investigated axon regeneration in DA9 neurons, using the ric-7(n2657) mutation as a background mutation. To sever the DA9 dorsal axon without damaging surrounding tissues, a pulsed laser was applied to a region of the axon near the presynaptic region (see the Materials and Methods section), and axon regrowth was assessed 24 and $48 \mathrm{~h}$ after axotomy.

Similar to PLM axon, DA9 axon regeneration was normal in the absence of esyt-2, but it was significantly reduced in the absence of jph-1 (Fig 7D and E). In control and esyt-2 mutant animals, the axon grew back to $\sim 150$ and $\sim 190 \mu \mathrm{m}$ in length from the cut-site at 24 and $48 \mathrm{~h}$, respectively. However, in jph-1 (ok2823) mutants, the axon regrew to $\sim 100$ and $\sim 150 \mu \mathrm{m}$ from the cut-site at 24 and $48 \mathrm{~h}$, respectively (Fig $7 D$ and $E$ ). These results suggest that proper function/assembly of neuronal ER-PM contacts is important for maintaining robust axon regeneration in DA9 neurons. We also examined the distribution of ER-PM contacts in regenerating axons. To this end, the distribution of ER-PM contacts was assessed $24 \mathrm{~h}$ after axotomy either by the split GFP approach or mNeonGreen:: ESYT-2. No significant changes in the distribution of ER-PM contacts were observed in axons or in dendrites (Fig S7A-D). These results indicate that the overall distribution of neuronal ER-PM contacts is maintained during axon regeneration after laser-induced injury.

The distribution of neuronal ER-PM contacts in cil-1 and atln-1 mutants is severely altered, and thus, it is possible that these contacts are functionally abnormal. To examine such possibility, we determined whether cil-1 and atln-1 mutants show any changes in resilience to axonal damage. Remarkably, similar to jph-1 (ok2823) mutants, cil-1(yas37) and atln-1(yas38) mutants exhibited significantly reduced axon regeneration, supporting the notion that ERPM contacts in these mutants are functionally abnormal. Even $48 \mathrm{~h}$ after axotomy, the axon was only $\sim 130 \mu \mathrm{m}$ from the cut-site in these mutants (Fig 7F and G). DA9-specific re-expression of CIL-1 in cil1(yas37) mutants and ATLN-1 in atln-1(yas38) mutants (via the itr$1 p B$ promoter) rescued these phenotypes, confirming that $\mathrm{CIL}-1$ and ATLN-1 function cell autonomously to maintain robust axon regeneration (Fig 7F and G).

These results suggest that proper distribution (non-uniform distribution) of neuronal ER-PM contacts is essential for maintaining robust axon regeneration. If this is indeed the case, restoring the distribution of neuronal ER-PM contacts alone should be sufficient to restore axon regeneration in cil-1(yas37) mutants. To test this hypothesis, we performed the laser axotomy experiment in

\footnotetext{
fluorescence intensity of reconstituted GFP (endogenous SEC-61 $\beta$ ) in dorsal axon of the same animals as in (G) (mean \pm SEM; Dunnett's multiple comparisons test; ${ }^{*} P=0.0468$ [Control versus ret-1 (tm390)], ${ }^{* *} P<0.0001$ [Control versus cil-1 (yas37)]). (I) Representative live SDC images of dendrite and dorsal axon of DA9 neurons, expressing mNeonGreen-tagged rough ER marker TRAP $\beta$ (TRAP $\beta:: m N e o n G r e e n)$ in animals with indicated genotype. Note the increased abundance of TRAP $\beta::$ mNeonGreen in both dendrite and dorsal axon in cil-1(yas37), ret-1(tm390), and atln-1(yas38) mutants. Scale bars, $20 \mu \mathrm{m}$. (J) Quantification of normalized fluorescence intensity of TRAP $\beta:: m$ NeonGreen in dendrite of DA9 neuron from wild-type control $(n=21)$, cil-1 (yas37) $(n=15)$, ret-1 (tm390) $(n=11)$, and atln-1 (yas38) ( $=17)($ mean \pm SEM; Dunnett's multiple comparisons test; ${ }^{*} P<0.0001$ [Control versus cil-1 (yas37) and Control versus ret-1 (tm390)], ${ }^{* *} P=0.0006[$ Control versus atln-1 (yas38)]). (K) The representative line scan profiles of TRAP $\beta:$ mNeonGreen along the dorsal axons of animals with indicated genotype.

Source data are available for this figure.
} 
A
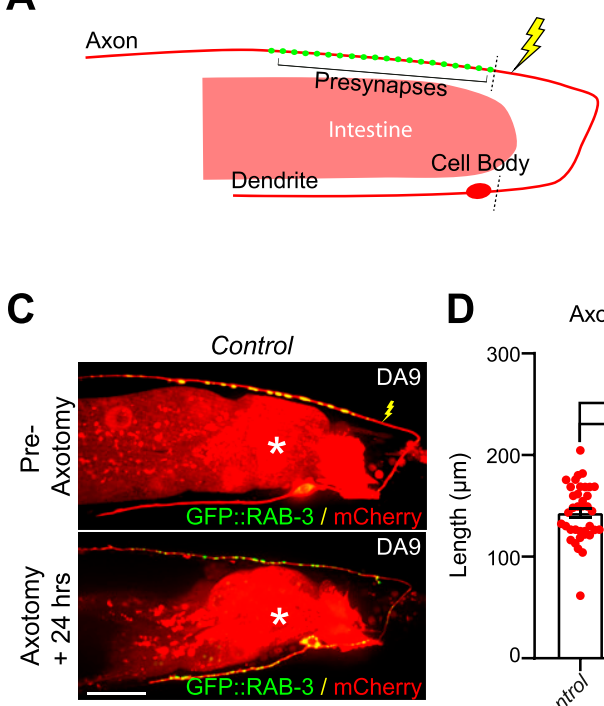

D Axotomy +24 hrs
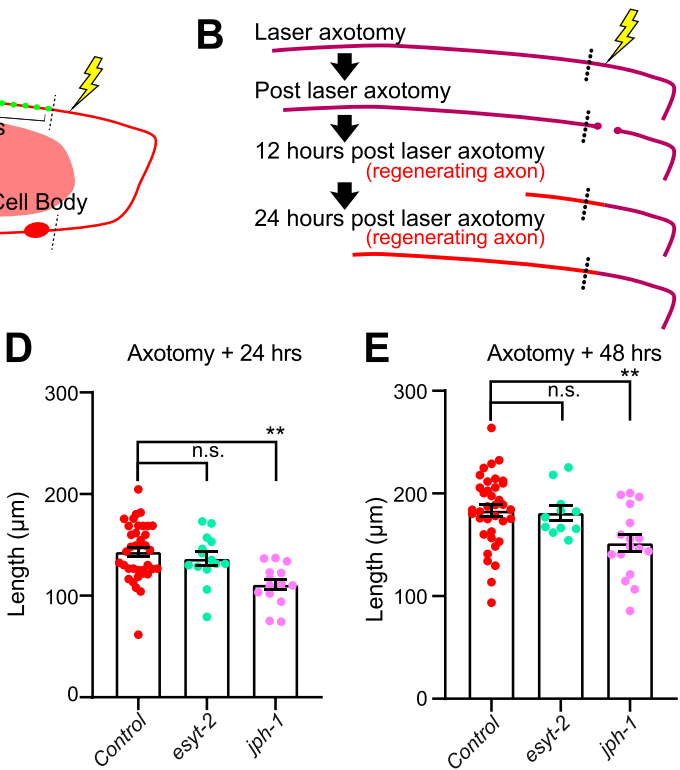

E

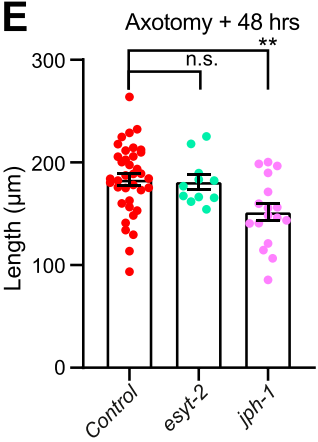

$\mathbf{F}$

Axotomy $+24 \mathrm{hrs}$

G

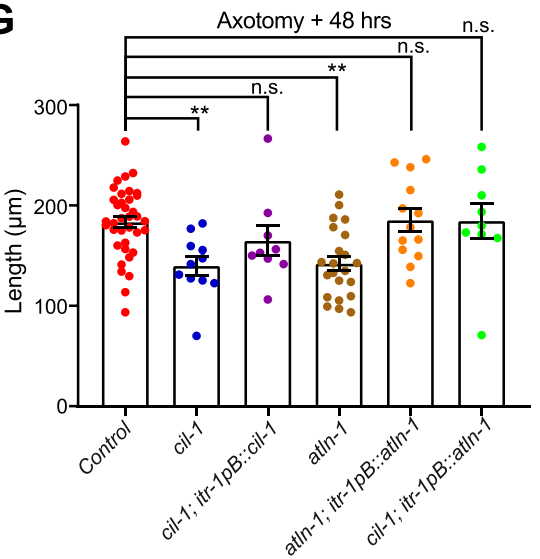

H
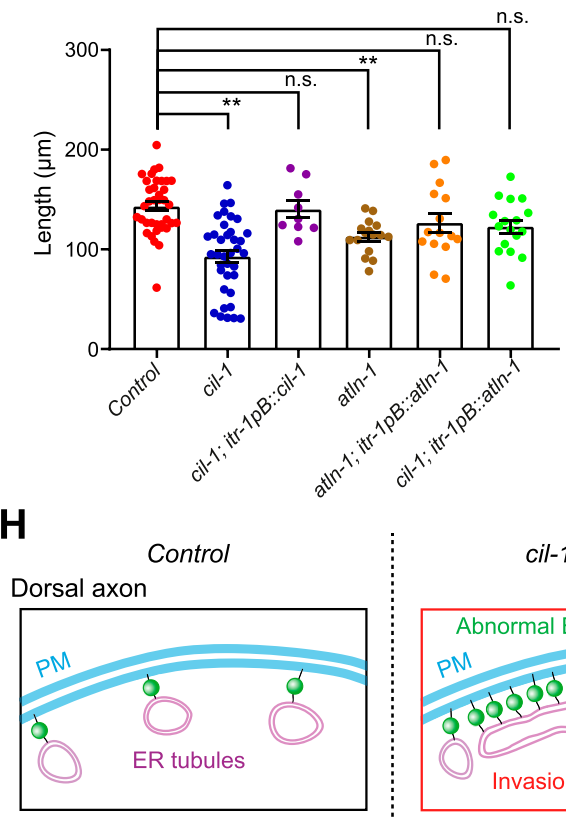

Regenerating axon

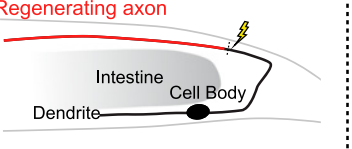

cil-1 or atln-1

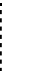

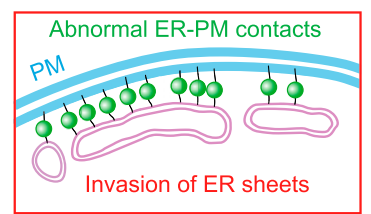

Shorter regenerating axon

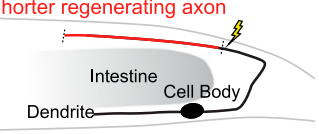

cil-1; ATLN-1 OE

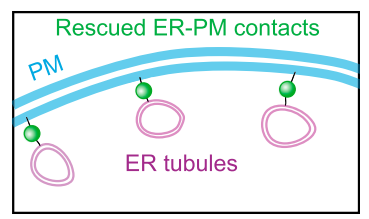

Rescued regenerating axon

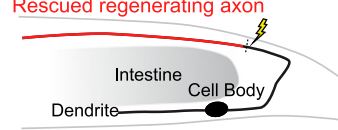

Figure 7. CIL-1 and ATLN-1 are both required for efficient axon regeneration.

(A) A schematic diagram showing the laser ablation site (a yellow lightning mark) proximal to the presynaptic region of DA9 neuron. (B) Cartoon depicting axon regeneration process at different time points after laser-induced axotomy. Red indicates newly regenerated axon after laser axotomy. Note that the axotomy was considered successful when two clear bulbs were observed at both ends of a severed axon. (c) Representative live spinning disc confocal images of DA9 neurons, co-expressing cytosolic soluble mCherry (red) and GFP::RAB-3 (green), from wild-type control animals before and $24 \mathrm{~h}$ after laser axotomy. The lightning marks denote the axotomy sites. The central autofluorescent region is the intestine (asterisk). Scale bar, $20 \mu \mathrm{m}$. (D, E) Quantification of regenerated DA9 dorsal axon length at $24 \mathrm{~h}$ (D) and $48 \mathrm{~h}$ (E) after laser axotomy in wild-type control and mutants as indicated. For $24 \mathrm{~h}$ as shown in (D): wild-type control ( $n=37)$, esyt-2 (syb709) ( $=14)$, and jph-1 (ok2823) $(n=15)$ (mean \pm SEM; Dunnett's multiple comparisons test; ${ }^{* \star} P=0.0002$ [Control versus jph-1(ok2823)], n.s. denotes not significant). For $48 \mathrm{~h}$ (E): wild-type control $(n=37)$, esyt-2 (syb709) $(n=11)$, and jph-1 (ok2823) $(n=17)$ (mean \pm SEM; Dunnett's multiple comparisons test; ${ }^{* *} P=0.0029$ [Control versus jph-1 (ok2823)], n.s. denotes not significant). Note the reduced axon regeneration compared to wild-type control in jph-1 (ok2823) mutants. (F, G) Quantification of regenerated DA9 dorsal axon length at $24 \mathrm{~h}$ (F) and $48 \mathrm{~h}$ (G) after laser axotomy in wild-type control and mutants as indicated. For $24 \mathrm{~h}$ (F): wild-type control ( $\mathrm{n}=37$, same control as in D), cil-1 (yas37) $(n=37)$, cil-1 (yas37); itr-1pB::cil-1 ( $=9)$, atln-1 (yas38) $(n=16)$, atln-1 (yas38); itr-1pB::atln-1 ( $=15)$, and cil-1 (yas37); itr-1pB:: atln-1 $(n=17)$ (mean \pm SEM; Dunnett's multiple comparisons test; ${ }^{* \star} P<0.0001$ [Control versus cil-1 (yas37)], ${ }^{* *} P=0.0057$ [Control versus atln-1 (yas38)], n.s. denotes not significant). For 48 h (G): wild-type control ( $n=37$, same control as in E), cil-1 (yas37) $(n=11)$, cil-1 (yas37); itr-1pB::cil-1 $(n=9)$, atln-1 (yas38) $(n=22)$, atln-1 (yas38); itr-1pB::atln-1 ( $n=13)$, and cil-1 (yas37); itr-1pB::atln-1 ( $n=9)$ (mean \pm SEM; Dunnett's multiple comparisons test; ${ }^{* *} P=0.0057$ [Control versus cil-1 (yas37)], ${ }^{* *} P=0.0005$ [Control versus atln-1 (yas38)], n.s. denotes not significant). (H) A model depicting the importance of the balance of ER sheets and tubules in maintaining the distribution of ER-PM contacts in DA9 neurons. In wild-type control animals, the axonal region is largely occupied with smooth tubular ER, whereas the somatodendritic region is occupied by both rough and smooth ER that consist of the network of ER sheets and tubules. The balance of ER tubules and sheets restrict the formation of neuronal ER-PM contacts primarily in somatodendritic region. In cil-1 and at $n-1$ mutants, ER sheets expand and invade into the dorsal axon as seen by ectopic presence of rough ER proteins in this region. This is accompanied by abnormal distribution of ER-PM contacts and disruption of their functions in neurons. Such

disruption, at least in part, contributes to reduced axon regeneration in these mutants. Supporting this notion, overexpression of ATLN-1 in cil-1 (yas37) mutants, which rescues the distribution of ER-PM contacts, is sufficient to restore axon regeneration.

Source data are available for this figure.

cil-1(yas37) mutants overexpressing ATLN-1 in DA9 neuron, which exhibited a normal distribution of ER-PM contacts despite the complete absence of CIL-1 (see Fig 5E-G). Overexpression of ATLN-1 in cil-1(yas37) mutants restored axon regeneration, as the axon regrew to $\sim 140$ and $\sim 190 \mu \mathrm{m}$ from the cut-site 24 and $48 \mathrm{~h}$ after axotomy, respectively, similar to controls (Fig $7 F$ and G).

Taken together, our results demonstrate the critical role of CIL-1 and ATLN-1 in axon regeneration following axonal damage and 
suggest the importance of maintaining the non-uniform distribution of neuronal ER-PM contacts in this process.

\section{Discussion}

In this study, we used the C. elegans motor neuron DA9 to understand how the distribution of neuronal ER-PM contacts is established and maintained. We showed that the distribution of neuronal ER-PM contacts critically depends on the activities of proteins that are involved in shaping the ER. Furthermore, they are essential for maintaining neuronal resilience against insults. Key findings of our current study are the following:

(1) We successfully visualized the distribution of ER-PM contacts in neurons in vivo using a split GFP approach and found that neuronal ER-PM contacts were non-uniformly distributed (enriched in somatodendritic regions and rare in axon) in C. elegans. Remarkably, some of these contacts contained the evolutionarily conserved ER-PM tethering protein, ESYT-2 (E-Syts).

(2) Using an unbiased forward genetic screen, we identified mutants that exhibited abnormal distribution of ER-PM contacts. Analysis of these mutants-namely, cil-1(yas37) and atln-1(yas38)-revealed the importance of cortical ER shape in maintaining the non-uniform distribution of ER-PM contacts in neurons. These mutants showed defects in ER tubule formation that was accompanied by expansion of cortical ER sheets and the ectopic formation of ER-PM contacts in dorsal axons.

(3) We found that the inositol 5-phosphatase, CIL-1 (human INPP5K), functioned at the ER, regulated the cortical ER network, and maintained the distribution of ER-PM contacts in neurons. In the absence of CIL-1, cortical ER sheets expanded with a concomitant reduction in ER tubules. Importantly, our results reveal that CIL-1 acts genetically upstream of ER-shaping ATLN-1 (human Atlastin-1). Overexpression of ATLN-1 could bypass the requirement of $\mathrm{CIL}-1$ to regulate cortical ER structure and maintain the distribution of ER-PM contacts, indicating their related functions.

(4) We found that the restriction of cortical ER sheets to somatodendritic regions was maintained by proteins that promote $E R$ tubule formation, including RET-1 (human reticulons), CIL-1, and ATLN-1. In the mutants with reduced functions of any of these proteins, rough ER sheets, which normally reside in the somatodendritic compartment, ectopically localized to the axonal compartment. Thus, the coordinated action of multiple ER-shaping proteins is critical for maintaining the identity of axonal ER and restricting cortical ER primarily to somatodendritic regions.

(5) Our results demonstrate the importance of CIL-1 and ATLN-1 in the resilience of neurons after neuronal damage. Using laser axotomy, we showed that efficient axon regeneration required the functions of these proteins. When the distribution of ER-PM contacts was restored in cil-1 mutants (by ATLN-1 overexpression), axon regeneration, which relies on normal function of ER-PM contacts (Kim et al, 2018), was also restored. This suggests that the normal distribution of ER-PM contacts is critical for the function of these contacts and for maintaining the ability of axons to regenerate after an insult (Fig $7 \mathrm{H})$.
Our results demonstrate that in the C. elegans DA9 neuron, the ER forms a network of sheets and tubules, similar to those seen in yeast and mammalian cells. Furthermore, we found that ER-PM contacts are most abundant in somatodendritic regions and scarce in the axon of this neuron. Similar to what we showed in the present study, previous highresolution $3 \mathrm{D}$ reconstruction of ER structure in mammalian neurons via electron microscopy has reported a non-uniform distribution of ER-PM contacts in different regions of the cell, with particular enrichment in the soma (Wu et al, 2017). Thus, the molecular mechanisms responsible for maintaining neuronal ER-PM contacts are likely evolutionarily conserved. Axonal ER is mostly tubular and smooth, whereas somatodendritic ER is a mixture of rough and smooth ER and consists of a network of both sheets and tubules (Wu et al, 2017; Yalcin et al, 2017; Terasaki, 2018; Farias et al, 2019). Although how exactly CIL-1/INPP5K and ATLN-1/Atlastin control the distribution of neuronal ER-PM contacts remains elusive, our study suggests that the balance between ER sheets and tubules may determine where ER-PM contacts are distributed in neurons based on the reported functions of INPP5K and Atlastin to regulate ER morphologies (see below). Axonal ER tubules may physically prevent ER-PM contacts from forming. In support of this notion, mutant worms lacking proteins involved in ER tubule formation, including RET-1/Reticulon, CIL1 , and ATLN-1, show ectopic formation of ER-PM contacts in the dorsal axon. We further provide evidence that smooth ER tubules in axons become more sheet-like with the ectopic presence of rough ER proteins in the reduced/eliminated activities of these proteins, resulting in the expansion of cortical ER sheets and ectopic formation of ER-PM contacts in axons (Fig 7H). We acknowledge the heterogeneity of ER-PM contacts. Because of the difficulty in determining precise ultrastructure of the ER by electron microscopy in C. elegans neurons, we used fluorescencebased detection of neuronal ER-PM contacts (i.e., the split GFP approach and ESYT-2). However, both the split GFP approach and ESYT-2 do not label all ER-PM contacts. Further studies are needed to examine whether the proteins that we investigated in the current study have general roles in maintaining the distribution of all types of ER-PM contacts.

Our genetic and cell biological data suggest that CIL-1 acts on the ER and regulates ATLN-1 activities. However, it is still not clear how CIL-1 function is related to ATLN-1 function. There is no strong evidence that supports the presence of $\mathrm{PI}(4,5) \mathrm{P}_{2}$ in ER membranes. It is possible that the presence of $\mathrm{CIL}-1 /$ INPP5K is the exact reason why $\mathrm{PI}(4,5) \mathrm{P}_{2}$ is not abundant in ER membranes; removal of $\mathrm{PI}(4,5) \mathrm{P}_{2}$ from ER membranes by CIL-1-dependent $\mathrm{PI}(4,5) \mathrm{P}_{2}$ dephosphorylation may be essential for ATLN-1 to regulate the balance of ER tubules and sheets. INPP5K was also reported to hydrolyze $\mathrm{PI}(3,4,5)$ $\mathrm{P}_{3}$, a phosphoinositide that is normally produced by phosphoinositide 3-kinase (PI3K) at the PM (Ijuin et al, 2000; Gurung et al, 2003; Schmid et al, 2004; Dong et al, 2018). Thus, whether PI(4,5) $\mathrm{P}_{2}$ is the major substrate of $\mathrm{CIL}-1$ deserves further investigation. Atlastins are primarily implicated in maintaining three-way ER junctions by promoting homotypic fusion of ER tubules. However, apart from its well-known function in homotypic ER fusion, there is evidence that Atlastins may play a role in ER tubule formation. First, Atlastins physically interact with curvature-stabilizing ER-shaping proteins, including reticulons and DP1 (Hu et al, 2009). Second, in yeast the depletion of Sey1p (the dynamin-like GTPase that resembles the Atlastin) together with Yop1p or Rtn1p results in loss of ER tubules and an increase in ER sheets (Hu et al, 2009; Anwar et al, 2012). Interestingly, the depletion of Sey1p together with Yop1p or Rtn1p 
also leads to the expansion of ER-PM contacts, as seen in other yeast mutants lacking ER tubule-shaping proteins (Rtn1p, Rtn2p, and DP1/Yop1p) (West et al, 2011). Thus, ATLN-1/Atlastins may also function to regulate ER tubule formation in certain circumstances. In fact, our newly isolated atln-1 reduction-of-function mutant showed a reduction in ER tubules and an expansion of cortical ER sheets, supporting this notion.

Our studies also provide novel insights into the mechanisms of ER domain segregation in neurons. Rough ER, which consists of ER sheets, localizes predominantly to the somatodendritic region but is generally absent from the dorsal axon in the DA9 neuron (Fig 6F). The lack of rough ER in most axonal processes has been reported for other $C$. elegans neurons (Rolls et al, 2002). Similar patterns of rough ER has also been seen in mammalian neurons, where rough ER localizes predominantly to the soma and dendrite, but is absent from the distal axon (Wu et al, 2017; Ozturk et al, 2020). Neurons are polarized cells, thus the limited presence of rough ER in the somatodendritic region is critical for the polarized transport of transmembrane and secreted proteins in neurons (Ramirez \& Couve, 2011; Cui-Wang et al, 2012). However, the mechanisms that regulate the segregation of rough and smooth ER domains in neurons has been a mystery. Using SEC-61 $\beta$ and TRAP $\beta$ as rough ER markers, we found that ER-shaping proteins play critical roles in restricting the distribution of rough ER proteins to the somatodendritic region in the DA9 neuron. In mutants with reduced/eliminated activities of ATLN-1, RET-1, or CIL-1, these proteins are no longer restricted to the somatodendritic region, invading into axonal ER. As ATLN-1, RET-1, and CIL-1 are all involved in ER tubule formation in neurons (as shown in the current study), the formation of smooth tubular ER in axons may normally prevent SEC-61 $\beta$ and TRAP $\beta$ (and potentially other rough ER proteins) from invading the axonal ER, contributing to the limited presence of rough ER in the somatodendritic region.

ESYT-2 localized to ER-PM contacts in the DA9 neuron, suggesting that E-Syts/ESYT-2 are involved in the function of neuronal ER-PM contacts. Although ESYT-2 is dispensable for axon regeneration in the DA9 (as shown in the current study) and PLM neurons in C. elegans (Kim et al, 2018), a recent study reported that E-Syts are required for the structure and function of ER-PM contact sites in Drosophila photoreceptors (Nath et al, 2020). In mice, however, depletion of all the three E-Syts does not result in major abnormalities (Sclip et al, 2016; Tremblay \& Moss, 2016). Thus, it is likely that ESYT-2 functions redundantly with other proteins, such as Junctophilin, at neuronal ER-PM contacts in C. elegans, although a recent study also suggested a non-cell autonomous function of Junctophilin in axon regeneration (Piggott et al, 2021). In yeast, tricalbins (yeast homologs of E-Syts) are distributed non-homogenously at cortical $E R$, localizing preferentially to highly curved regions of cortical ER sheets (Collado et al, 2019; Hoffmann et al, 2019). In this organism, ER shape contributes to the organization of ER-PM contacts into compositionally distinct subdomains, positioning tricalbins and other ER-anchored proteins within distinct regions of the cortical ER (Hoffmann et al, 2019). Whether the organization of cortical ER subdomains is altered in C. elegans mutants with reduced/ eliminated activities of CIL-1, ATLN-1, or RET-1 needs further investigation. Importantly, E-Syt-mediated ER-PM tethering, as well as lipid transport, are both controlled by levels of cytosolic $\mathrm{Ca}^{2+}$ (Chang et al, 2013; Giordano et al, 2013; Fernandez-Busnadiego et al, 2015; Idevall-Hagren et al, 2015; Saheki et al, 2016; Bian et al, 2018).
Thus, cytosolic $\mathrm{Ca}^{2+}$-dependent regulation of the properties of neuronal ER-PM contacts also deserves further investigation.

Finally, our study suggests that the unique distribution of neuronal ERPM contacts is associated with the resilience of neurons against neuronal damage. Laser axotomy experiments revealed that axon regeneration is significantly reduced in mutant worms with reduced/eliminated activities of CIL-1 or ATLN-1 compared to controls. Mutants lacking CIL-1 or ATLN-1 show ectopic formation of axonal ER-PM contacts. Petkovic et al (2014), suggested that lipid delivery at neuronal ER-PM contacts promote neurite outgrowth (Petkovic et al, 2014). Thus, proper compositions of ER-PM contacts rather than their abundance may be important for regeneration or outgrowth of axons. Our study does not rule out the possibility that the axonal ER morphology (i.e., tubular versus sheet) and motility as well as other related functions of the axonal ER, such as maintenance of $\mathrm{Ca}^{2+}$ homeostasis and regulation of the functions of various other organelle contact sites, may also play a role in proper axon regeneration. INPP5K, a human homolog of $\mathrm{CIL}-1$, is highly expressed in the developing and adult brain, eye, muscle, and kidney (ljuin et al, 2008). Interestingly, overexpression of INPP5K has been shown to promote axon regeneration in mammalian neurons (Fink et al, 2017). Human bi-allelic point mutations that impair INPP5K's phosphatase activity lead to congenital muscular dystrophy with additional clinical manifestations that include cataracts, short stature, and intellectual impairments (Wiessner et al, 2017). Mutations in a number of wellestablished ER-shaping proteins, such as ATL1 (SPG3A) and RTN2 (SPG12), cause hereditary spastic paraplegia, a syndrome characterized by progressive weakness and spasticity mainly caused by the degeneration of motor and sensory axons (Blackstone, 2012). More recently, autosomal-dominant missense mutations in ATL3 were reported to cause hereditary sensory and autonomic neuropathy (HSAN) (Fischer et al, 2014; Kornak et al, 2014). These mutations disrupt fusion of the ER and induce aberrant ER tethering, causing major changes in ER morphology. Interestingly, cells carrying a disease-causing ATL3 mutation show pleiotropic defects in ER-related functions, including defects in vesicular transport and autophagosome formation (Krols et al, 2018, 2019; Behrendt et al, 2019). Our study shows that CIL-1/INPP5K and ATLN-1/ Atlastin-1 help to maintain the non-uniform distribution of neuronal ERPM contacts. These proteins likely contribute to the maintenance of the unique distribution of neuronal ER-PM contacts through their properties to control ER morphology, although we do not exclude the possibility that they control the distribution of ER-PM contacts indirectly through the regulation of other ER-related functions. Maintenance of the distribution of neuronal ER-PM contacts may be essential for the normal development and resilience of neurons in humans. Whether the development and progression of various neurological disorders is associated with the abnormal distribution and function of neuronal ER-PM contacts deserves further investigation.

\section{Materials and Methods}

\section{C. elegans strains and maintenance}

All C. elegans strains were maintained under standard conditions at room temperature $\left(22^{\circ} \mathrm{C}-24^{\circ} \mathrm{C}\right)$ on nematode growth media (NGM) agar plates seeded with the bacterial Escherichia coli (E. coli) strain 
OP50 as a food source, as previously described (Brenner, 1974). Wild-type was N2 Bristol strain. All experiments were performed on L4 larva hermaphrodite animals at room temperature, unless stated otherwise. Germline transformation was carried out as described (Mello \& Fire, 1995). Some strains were provided by the Caenorhabditis Genetics Center and the National Bioresource Project. Mutants and balancers used were jph-1(ok2823) I, cil-1(my15) III, ret-1(tm390) V, tmC25 [unc-5(tm/s1241)] IV, qC1 [dpy-19(e1259) glp-1(q339)] nIs189 III.

\section{Molecular cloning and strains}

Primers and plasmids used in this study are listed in Table S1. Strains used in this study are listed in Table S2.

\section{For C. elegans expression}

Cloning of itr-1pB::CP450::splitGFP1-10 Genomic DNA fragment corresponding to itr-1pB was ligated into odr-3p::CP450::mCherry (Saheki \& Bargmann, 2009) in the Fsel and Ascl sites, to generate itr1pB::CP450::mCherry. CDNA corresponding to splitGFP1-10 (see below) was PCR amplified and ligated into itr-1PB::CP450::mCherry in the Xhol and EcoRl sites, using the following primer set, splitGFP1-10_Xhol_F and splitGFP1-10_ECORL_R, to generate itr-1pB::CP450::splitGFP1-10.

CDNA encoding codon optimized splitGFP1-10 with synthetic introns (lower case) for C. elegans expression:

ATGTCCAAGGGAGAGGAGCTTTTCACCGGAGTCGTCCCAATCCTTGTCGA GCTTGACGGAGACGTCAACGGACACAAGTTCTCCGTCCGCGGAGAGGGAGA GGGAGACGCCACCATCGGAAAGCTTACCCTTAAGTTCATCTGCACCACCGGA AAGCTTCCAGTCCCATGGCCAACCCTTGTCACCACCCTTACCTACGGAGTCCA ATGCTTCTCCCGCTACCCAGACCACATGAAGCGCCACGACTTCTTCAAGTCCG CCATGCCAGAGGGATACGTCCAAGAGCGCACCATCTCCTTCAAGgtaagtttaa acatatatatactaactactgattatttaaattttcagGACGACGGAAAGTACAAGACCC GCGCCGTCGTCAAGTTCGAGGGAGACACCCTTGTCAACCGCATCGAGCTTAA GgtaagtttaaacagttcggtactaactaaccatacatatttaaattttcagGGAACCGACT TCAAGGAGGACGGAAACATCCTTGGACACAAGCTTGAGTACAACTTCAACTC CCACAACGTCTACATCACCGCCGACAAGCAGAAGAACGGAATCAAGGCCAAC TTCACCgtaagtttaaacatgattttactaactaactaatctgatttaaattttcagGTCCGC CACAACGTCGAGGACGGATCCGTCCAACTTGCCGACCACTACCAGCAAAACA CCCCAATCGGAGACGGACCAGTCCTTCTTCCAGACAACCACTACCTTTCCACC CAAACCGTCCTTTCCAAGGACCCAAACGAGAAG.

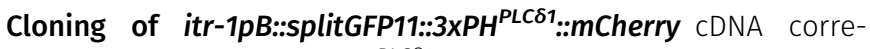
sponding to splitGFP11_3XPH ${ }^{P L C \delta 1}$ was PCR amplified and ligated in the Nhel and Kpnl sites of itr-1pB::CP450::mCherry, using a gBlock (IDT) and the following primer set, splitGFP11_3xPH-PLC81_Nhel_F and splitGFP11_3xPH-PLC81_Kpnl_R, to generate itr-1pB::splitGFP11:: $3 \times P H^{P L C \delta 1}:: m$ Cherry.

gBlock encoding codon optimized splitGFP11_ $3 \times \mathrm{PH}^{\mathrm{PLC} \delta 1}$ for $\mathrm{C}$. elegans expression (cDNA encoding codon optimized splitGFP11 is underlined):

ATGCGAGATCATATGGTTCTGCACGAATACGTCAACGCTGCCGGCATTAC AGGTGGCAAATTCATGTGTACAAGAGATCTtGAGCTCAAGCTTCACGGCCTGC AAGACGATCCGGATCTTCAGGCCTTGTTAAAAGGCTCGCAACTGTTAAAAGTC AAGTCATCATCTTGGCGAAGAGAGAGATTTTACAAATTACAGGAAGACTGTA AAACCATATGGCAAGAGTCTCGAAAGGTTATGAGATCCCCTGAATCCCAGCT GTTTTCGATAGAAGACATTCAAGAGGTACGTATGGGCCACCGTACTGAGGGA TTGGAAAAATTTGCTCGTGACATACCGGAGGATAGATGCTTTTCAATTGTTTT
CAAAGACCAAAGAAACACGCTTGACCTCATTGCGCCGTCGCCTGCTGATGCG CAACACTGGGTCCAGGGCCTCAGAAAAATAATACATCATTCTGGCTCGATGG ATCAGAGACAAAAGGGTGGTTCTGGTGGCCACGGCTTGCAGGACGACCCTGA CCTCCAAGCACTGTTAAAGGGCTCTCAGTTGTTAAAAGTAAAATCATCCAGTT GGAGACGAGAGAGATTTTATAAACTTCAGGAAGATTGTAAGACCATCTGGCA AGAATCTAGAAAAGTCATGCGATCGCCAGAATCTCAGCTCTTTTCGATTGAGG ATATTCAGGAAGTGAGAATGGGCCACCGAACAGAGGGATTGGAGAAATTTGC CCGAGATATTCCGGAAGATCGTTGTTTTTCAATTGTGTTTAAGGATCAAAGAA ATACCTTAGACTTGATAGCTCCTTCCCCAGCCGATGCTCAGCACTGGGTACAG GGTCTCCGAAAAATCATCCATCATTCGGGCTCTATGGACCAACGTCAAAAAG GTGGAAGTGGTGGTCATGGCCTTCAAGATGATCCGGACCTCCAGGCGTTACT CAAGGGTTCTCAATTGCTGAAAGTGAAATCGTCTAGTTGGAGAAGAGAAAGA TTCTATAAACTTCAAGAGGACTGCAAGACAATCTGGCAAGAGAGTCGTAAGG TTATGCGTTCGCCTGAGTCTCAACTGTTCAGTATCGAGGATATCCAAGAGGTG CGAATGGGTCACCGAACCGAGGGCCTGGAGAAGTTCGCGCGAGACATCCCT GAGGATCGATGTTTCTCAATTGTGTTTAAAGATCAGCGTAATACCCTGGATCT TATTGCACCGAGTCCGGCGGATGCACAGCACTGGGTTCAAGGTCTCAGAAAG ATTATACACCATTCTGGTTCAATGGATCAAAGACAGAAGCA.

Cloning of itr-1pB::splitGFP11::3XPH ${ }^{P L C 81}$ cDNA corresponding to splitGFP11_3XPH ${ }^{P L C \delta 1}$ was PCR amplified and ligated in the Nhel and EcoRl sites of itr-1pB:::CP450::mCherry, using a gBlock (IDT) (see above) and the following primer set, splitGFP11_3XPH-PLC81_Nhe1_F and splitGFP11_3XPH-PLC81_ECoRL_R, to generate itr-1pB::splitGFP11:: $3 X P H^{P L C \delta 1}$.

Cloning of itr-1pB::mNeonGreen::ESYT-2 Genomic DNA fragment corresponding to itr-1pB was ligated into esyt-2p::mNeonGreen:::ESYT2 in the Fsel and Ascl sites, to generate itr-1pB::mNeonGreen::ESYT-2.

Cloning of itr-1pB::wrmScarlet::ESYT-2 cDNA corresponding to wrmScarlet (El Mouridi et al, 2017) was PCR amplified and ligated into the itr-1pB::mNeonGreen::ESYT-2 in the Ascl and EcoRl sites, using the following primer set, wrmScarlet_Ascl_F and wrmScarlet_EcoRl_R, to generate itr-1pB:::wrmScarlet::ESYT-2.

Cloning of itr-1pB::splitGFP11::mCherry cDNA corresponding to $3 \times \mathrm{PH}^{\mathrm{PLC} \delta 1}$ was deleted by site-directed mutagenesis in itr-1pB: splitGFP11::3XPH ${ }^{P L C \delta 1:: m C h e r r y, ~ u s i n g ~ t h e ~ f o l l o w i n g ~ p r i m e r ~ s e t, ~}$ splitGFP11_mutagenesis_F and splitGFP11_mutagenesis_R, to generate itr-1pB::splitGFP11::mCherry.

Cloning of itr-1pB::FLP and odr-3p::FLP Genomic DNA fragment corresponding to itr-1PB was PCR amplified and ligated into the pMLS262 vector in the Avrll and Fsel sites, using C. elegans genomic DNA as a template and the following primer set, pMLS262_itr1pB_Avrll_F and pMLS262_itr-1pB_Fsel_R, to generate itr-1pB::FLP.

Genomic DNA fragment corresponding to odr-3p was PCR amplified and ligated into the pMLS262 vector in the Avrll and Fsel sites, using C. elegans genomic DNA as a template and the following primer set, pMLS262_odr3p_Avrll_F and pMLS262_odr-3p_Fsel_R, to generate odr-3p::FLP.

Cloning of itr-1pB::cil-1 cDNA of cil-1 (C50C3.7) was PCR amplified and ligated into itr-1pB:.jph-1::mCherry in the Nhel and Kpnl sites, using $C$. elegans CDNA library as a template and the following primer set, cil1_Nhel_F and cil-1_stop_Kpnl_R, to generate itr-1pB.:cil-1. Stop codon was placed between cil-1 cDNA and mCherry to prevent mCherry expression. 
Cloning of itr-1pB::cil-1 ${ }^{\text {N175A }}$ The amino-acid present in the phosphatase domain of $\mathrm{CIL}-1$ that was reported to be essential for its catalytic activity (N175) (Bae et al, 2009) was mutated to alanine using site-directed mutagenesis in itr-1pB::cil-1 with the primer set, cil-1_N175A_mutagenesis_F and cil-1_N175A_mutagenesis_R, to generate itr-1pB:.:cil-1 ${ }^{\mathrm{N175A}}$.

Cloning of itr-1pB::cil-1 ${ }^{\Delta S K I C H}$ Genomic DNA fragment corresponding to itr-1pB was ligated into pSM_SL2_mCherry (Pokala et al, 2014) in the Fsel and Ascl sites, to generate itr-1pB::SL2_mCherry. cDNA corresponding to $\mathrm{CIL}-1$ lacking the SKICH domain (amino acids 1-284 of CIL-1) was PCR amplified and ligated into itr-1pB:.:SL2_mCherry in the Nhel and Kpnl sites, using itr-1pB::cil-1 as a template and the following primer set, cil-1_Nhel_F and cil-1_ASKICH_Kpn1_R, to generate itr-1pB:: cil-1 ${ }^{\triangle S K I C H}$

Cloning of itr-1pB::cil-1 ${ }^{\text {PM }}$ cDNA corresponding to the 5-phosphatase domain of $C$. elegans cil- 1 was PCR amplified using the following primer sets, Kpnl-cil-1_S and BamHI-stop-cil-1_AS. The PCR products were then ligated at KpnI and BamHI sites in the LckmScarlet-I vector to generate mScarlet-I-CIL-1 ${ }^{P M}$. Lck-mScarlet-I (mScarlet-I ${ }^{P M}$ control for mammalian expression) was a gift from Dorus Gadella (plasmid \# 98821; Addgene). Genomic DNA fragment corresponding to itr-1pB was ligated into odr-3:: cb5:::mCherry (Saheki \& Bargmann, 2009) in the Fsel and Ascl sites, to generate itr-1pB::cb5:: mCherry. cDNA corresponding to Lck-mScarlet-I-CIL was then PCR amplified from mScarlet-I-CIL-1 ${ }^{\mathrm{PM}}$ using the following primer set, Lck_mScarlet_5ptase_CIL-1_F and Lck_mScarlet_5ptase_CIL-1_R and ligated into itr-pB:::cb5::mCherry via the ligation-independent cloning method to generate itr-1pB::cil-1 ${ }^{P M}$.

Cloning of itr-1pB::cil-1 ${ }^{E R}$ cDNA corresponding to CP450 was PCR amplified and ligated into itr-1pB:: $c i l-1^{\triangle S K I C H}$ in the Ascl and Nhel sites, using odr-3p:::CP450::mCherry (Saheki \& Bargmann, 2009) as a template and the following primer set, CP450_Ascl_F and CP450_Nhel_R, to generate itr-1pB:.:cil-1 ${ }^{E R}$.

Cloning of itr-1pB::TRAPbeta::mNeonGreen CDNA of trap-2 (T04G9.5) was PCR amplified and ligated into itr-1pB:::CP450::mNeonGreen in the Ascl and Smal sites, using C. elegans cDNA library as a template and the following primer set, TRAPbeta_T04G9.5_Ascl_F and TRAPbeta_T04G9.5_Smal_R, to generate itr-1pB::TRAPbeta::mNeonGreen.

Cloning of mig-13p::split-GFP11::mCherry Genomic DNA corresponding to mig-13p was PCR amplified and ligated into itr-1pB:: splitGFP11::mCherry in the Fsel and Ascl sites, using C. elegans genomic DNA as a template and the following primer set, mig-13p_Fsel_F and mig-13p_Ascl_R, to generate mig-13p::split GFP11::mCherry.

Cloning of itr-1pB::atln-1 cDNA of atln-1 (Y54G2A.2a) was PCR amplified and ligated into itr-1pB::jph-1::mCherry in the Ascl and Kpnl sites, using C. elegans cDNA library as a template and the following primer set, atln-1_Ascl_F and atln-1_stop_KpnI_R, to generate itr-1pB::atln-1. Stop codon was placed between atln-1 cDNA and mCherry to prevent mCherry expression.

Cloning of itr-1pB::atln-1 ${ }^{\text {E338K }}$ E338K mutation in ATLN-1 [a mutation mimicking atln-1(yas38)] was introduced using site-directed mutagenesis in itr-1pB::atln-1 with the primer set, atln-1_E338K_site mutation_F and atln-1_E338K_site mutation_R, to generate itr-1pB:: atln-1 $1^{\mathrm{E3} 38 \mathrm{~K}}$.

Cloning of itr-1pB::atln-1 ${ }^{\text {K80A }}$ K80A mutation in ATLN-1 (a mutation that makes the GTP-binding activity of ATLN-1 defective [Liu et al, 2019]) was introduced using site-directed mutagenesis in itr-1pB:: atln-1 with the primer set atln-1_K80A_site mutation_F and atln1_K80A_site mutation_R, to generate itr-1pB::atln-1 ${ }^{\text {K80A }}$.

Cloning of itr-1pB::CP450::mCherry Genomic DNA fragment corresponding to itr-1pB was ligated into odr-3::CP450::mCherry (Saheki \& Bargmann, 2009) in the Fsel and Ascl sites, to generate itr-1pB:: CP450::mCherry.

Cloning of itr-1pB::ocrl-1 ${ }^{E R}$ cDNA corresponding to ocrl-1 5-phosphatase domain was PCR amplified and ligated into itr-1pB::cil-1 ${ }^{E R}$ in the Nhel and Xhol sites, using $C$. elegans cDNA library as a template and the following primer set, ocrl-1_IPPC_Nhel-F and ocrl1_IPPC_Xhol-R, to generate itr-1pB::Ocrl-1 ${ }^{E R}$.

Cloning of itr-1pB::unc-26 ${ }^{E R}$ CDNA corresponding to unc-26 5phosphatase domain was PCR amplified and ligated into itr-1pB::cil$1^{E R}$ in the Nhel and Kpnl sites, using C. elegans cDNA library as a template and the following primer set, unc-26_IPPC_Nhel-F and unc-26_IPPC_Kpnl-R, to generate itr-1pB::unc-26 ${ }^{E R}$.

Cloning of itr-1pB::ocrl-1 ${ }^{P M}$ CDNA corresponding to Lck-mScarlet-IOCRL-1 was PCR amplified from mScarlet-I-OCRL-1PM using the following primer set, Lck_mScarlet_5ptase_CIL-1_F and Lck_mScarlet_5ptase_OCRL1_R and ligated into itr-pB:.:cb5::mCherry via the ligation-independent cloning method to generate itr-1pB::ocrl- ${ }^{\mathrm{PM}}$.

Cloning of itr-1pB::unc-26 ${ }^{P M}$ cDNA corresponding to Lck-mScarletI-UNC-26 was PCR amplified from mScarlet-I-UNC-26 $6^{P M}$ using the following primer set, Lck_mScarlet_5ptase_CIL-1_F and Lck_mScarlet_5ptase_UNC26_R and ligated into itr-pB.:cb5:.:mCherry via the ligation-independent cloning method to generate itr-1pB::unc-26 ${ }^{P M}$.

Cloning of itr-1pB::mCherry::CLIMP-63 CDNA corresponding to CLIMP-63 was PCR amplified and ligated into itr-1pB::m $m$ Cherry::JPH-1 in the Nhel and Kpnl sites, using CLIMP-63 pcDNA (plasmid \#80977; Addgene) as a template and the following primer set, CLIMP-63_NhelF and CLIMP-63_KpnI-R, to generate itr-1pB::mCherry::CLIMP-63.

\section{For mammalian expression}

Cloning of OCRL-1 ${ }^{\mathrm{ER}}$-EGFP and UNC-26 ${ }^{\mathrm{ER}}$-EGFP CDNA corresponding to ER-targeted 5-phosphatase domain of C. elegans ocrl-1 [itr-1pB::ocrl-1 ${ }^{E R}$ ] or unc-26 [itr-1pB::unc-26 $6^{E R}$ ] was PCR amplified using the following primer sets, Xhol-CP450_S and Apal-ocrl1_AS for OCRL-1 ${ }^{\text {ER }}$; Xhol-CP450_S and Apal-unc-26_AS for UNC-26 ${ }^{\text {ER }}$. The PCR products were then ligated at Xhol and Apal sites in pEGFP-N1 (EGFP control) to generate OCRL-1 ${ }^{E R}-E G F P$ and UNC-26 ER $-E G F P$.

Cloning of mScarlet-I-OCRL-1 ${ }^{\mathrm{PM}}$ and mScarlet-I-UNC-26 ${ }^{\mathrm{PM}}$ CDNA corresponding to the 5-phosphatase domain of C. elegans ocrl-1 or unc-26 was PCR amplified using the following primer sets, BsrGl-ocrl1_S 
and BamHI-stop-ocrl1_AS for OCRL-1 ${ }^{\text {PM }}$, Kpnl_unc-26_S and Bglll-stop-unc26_AS for UNC-26 ${ }^{\mathrm{PM}}$. The PCR products were then digested with BsrGI and BamHI for OCRL-1 ${ }^{\mathrm{PM}}$ or Kpnl and Bglll for UNC-26 ${ }^{\mathrm{PM}}$ and ligated at Acc65l and BamHI sites or Kpnl and BamHI sites in the Lck-mScarlet-I vector to generate $m S$ carlet-I-OCRL-1 ${ }^{P M}$ and $m S$ carlet-I-UNC-26 ${ }^{P M}$, respectively.

\section{Isolation and characterization of cil-1 (yas37) and atln-1 (yas38)}

A strain expressing mNeonGreen:ESYT-2 in DA9 neuron (sybls50) was mutagenized with EMS according to standard protocols (Anderson, 1995). 1000 F1s were cloned into different plates, and 30-50 F2 animals from individual F1 animals were subjected to a direct visual screen under a microscope. The mutants were isolated based on the changes in mNeonGreen:: ESYT-2 distribution in DA9 neuron as observed with a 40x objective on either a Leica DMi8 microscope or a Nikon Ti2 inverted microscope.

\section{Mapping of cil-1 and atln-1}

cil-1(yas37) and atln-1(yas38) were mapped to chromosome III and chromosome IV, respectively, using balancer strains (Dejima et al, 2018). Genomic DNA of the mutants were extracted and sent to GENEWIZ, Inc. for next generation sequencing. CDNA encoding CIL-1 was PCR amplified and ligated into a vector that contains DA9 specific itr-1PB promoter and injected at $5 \mathrm{ng} / \mu \mathrm{l}$ into cil-1(yas37) mutants; CDNA encoding ATLN-1 was PCR amplified and ligated into a vector that contains DA9 specific itr-1pB promoter and injected at $5 \mathrm{ng} / \mu \mathrm{l}$ into at $n$-1(yas38) mutants. The constructs rescued mNeonGreen::ESYT-2 localization in DA9 neurons. To identify the cil1 and atln-1 mutations, the cil-1 genomic coding region in yas 37 and at $n-1$ genomic region coding yas 38 were amplified by PCR, and PCR products were sequenced.

\section{Cell-type specific endogenous tagging of ESYT-2 by CRISPR/cas9}

A cell-specific CRISPR protocol, a SapTrap approach (Schwartz \& Jorgensen, 2016), was used to endogenously tag GFP to the $N$ terminus of ESYT-2 in specific neurons. Upon injection of FLPase, the off cassette was removed, leaving the $\mathrm{N}$-terminal GFP and a single FRT site that encodes a 12-amino-acid flexible linker (GSSYSLESIGTS) in front of the endogenous ESYT-2. To achieve DA9 or AWC specific expression of endogenously tagged GFP::ESYT-2 (endoGFP:: ESYT-2), FLPase was expressed under DA9-selective itr-1pB promoter or AWC-selective odr-3 promoter.

\section{Generation of esyt-2 knock-out strain}

Knock-out allele of esyt-2 (syb709) was generated by SunyBiotech Corporation using CRISPR/Cas9 genome editing method. 1,902 bp ( $627 \mathrm{bp}-2,528 \mathrm{bp}$ ) of esyt-2 were deleted from the respective gene of N2 worms. The strain was backcrossed 6 times to N2 before use.

\section{Endogenous tagging of SEC-61.B with splitGFP1-10}

sec-61.B (knu560) was generated by Knudra Transgenics. cDNA encoding splitGFP1-10 was inserted before the initiation codon of SEC-61.B to generate N-terminal splitGFP1-10-tagged SEC-61.B. The strain was backcrossed six times to N2 before use.

\section{Brood size assay}

Brood size assay was conducted for control N2 and atln-1 (yas38) animals at $20^{\circ} \mathrm{C}$. $\mathrm{L} 4$ worms of $\mathrm{N} 2$ and atln-1 (yas38) were singled out to NGM plates that had been seeded with OP50. The number of eggs that are laid and hatched were counted manually for the next three consecutive days. The number of progeny laid during the three consecutive days were plotted against strain type (Fig S3D). The average number of eggs laid and hatched for each strain per day for each strain was plotted against the day number (Fig S3E). At least eight independent plates for each strain were assayed.

\section{Cell culture and transfection}

HeLa cells were cultured in DMEM containing 10\% FBS and 1\% penicillin/streptomycin at $37^{\circ} \mathrm{C}$ and $5 \% \mathrm{CO}_{2}$. Transfection of plasmids was carried out with Lipofectamine 2000 (Thermo Fisher Scientific). Cells were routinely verified as free of mycoplasma contamination at least every 2 mo, using MycoGuard Mycoplasma PCR Detection Kit (Genecopoeia). No cell lines used in this study were found in the database of commonly misidentified cell lines that is maintained by ICLAC and NCBI Biosample.

\section{Live imaging by fluorescence microscopy}

L4 hermaphrodite animals with fluorescently tagged proteins were transferred to a glass slide and immobilized on 3\% agarose pads using 2-3 $\mu \mathrm{l} 1 \mathrm{mg} / \mu \mathrm{l}$ levamisole diluted in M9 buffer. Multiple transgenic lines of each transgene were examined for fluorescent expression and localization patterns. HeLa cells were washed twice and incubated with $\mathrm{Ca}^{2+}$ containing buffer $(140 \mathrm{mM} \mathrm{NaCl}, 5 \mathrm{mM} \mathrm{KCl}, 1$ $\mathrm{mM} \mathrm{MgCl}, 10 \mathrm{mM}$ Hepes, $10 \mathrm{mM}$ glucose, and $2 \mathrm{mM} \mathrm{CaCl}_{2}, \mathrm{pH} 7.4$ ) before imaging. All images were captured under a 100× objective.

SDC microscopy and super-resolution SDC-structured illumination microscopy (SDC-SIM) were performed on a setup built around a Nikon Ti2 inverted microscope equipped with a Yokogawa CSU-W1 confocal spinning head, a Plan-Apo objective (100 × 1.45 NA), a back-illuminated sCMOS camera (Prime 95B; Photometrics), and a super-resolution module (Live-SR; Gataca Systems) that was based on structured illumination with optical reassignment and image processing (Roth \& Heintzmann, 2016). The method, known as multifocal structured illumination microscopy (York et al, 2012), makes it possible to double the resolution and the optical sectioning capability of confocal microscopy simultaneously. The maximum resolution is $128 \mathrm{~nm}$ with a pixel size in super-resolution mode of $64 \mathrm{~nm}$. Excitation light was provided by $488 \mathrm{~nm} / 150 \mathrm{~mW}$ (Coherent) (for GFP/mNeonGreen) and $561 \mathrm{~nm} / 100 \mathrm{~mW}$ (Coherent) (for mCherry/wrmScarlet/RFP/ mScarlet-I) and 647-nm/125 mW (for iRFP) (power measured at optical fiber end) DPSS laser combiner (iLAS system; Gataca systems). All image acquisition and processings were controlled by MetaMorph (Molecular Device) software. Images were acquired with exposure times in the 400-500 ms range.

\section{Laser axotomy}

Axotomy experiments were performed as described previously (Hammarlund et al, 2009). Strains were crossed into XE1931, a kind gift from Marc Hammarlund, before performing axotomy. Before 
laser ablation experiments, 14 animals were immobilized without anesthetics by using $0.1 \mu \mathrm{m}$ diameter polystyrene microsphere polybeads (Polysciences) and mounted onto $3 \%$ agarose pads on a glass slide. UV ablation was carried out on a GATACA iPulse system based on a dual galvanometer scanning system. After laser equipment calibration, DA9 neurons were cut at the posterior part of the asynaptic region using $355 \mathrm{~nm}$ pulsed laser. Animals were then recovered to OP50-seeded NGM plates and scored later with SDC microscopy.

\section{Image analysis and quantification}

All images were analyzed off-line using Fiji (http://fiji.sc/wiki/ index.php/Fiji). Quantification of fluorescence signals was performed using Excel (Microsoft) and Prism 7 or 8 (GraphPad Software).

\section{Quantification of ER-PM contacts, PM marker, and ER marker}

For quantification of ER-PM contacts, PM marker and ER marker that were visualized by the split-GFP approach (extrachromosomal array for Figs $1 \mathrm{G}$ and S1C and integrated array for Figs 4I, 5D, 6E, and S7B), $z$-stack images of the side view of a DA9 neuron were taken by SDC microscopy and were projected into a single plane by maximum projection. Background fluorescence was measured in a region close to a DA9 neuron and subtracted from each maximum projected image. The background-subtracted image was then converted to a 10-bit image, and the DA9 neuron was straightened using the "Straighten" function. Regions of $40 \mu \mathrm{m}$ in width from the start of dendrite (for dendrite) and from the start of dorsal axon (for dorsal axon) were selected for analysis. The clusters/particles containing mCherry (for PM marker) or reconstituted GFP signals (for ER-PM contacts and ER marker) above an arbitrary threshold were segmented, and area and mean fluorescent intensity of individual clusters/particles were measured using the "Analyze particles" command. The total fluorescence intensity was calculated as a sum of the product of the area and mean fluorescent intensity of individual clusters/particles, and then, the ratio of total fluorescence intensity of dorsal axon to that of dendrite was plotted.

\section{Quantification of endoGFP::ESYT-2}

For quantification of endoGFP::ESYT-2 (Fig 2C), worms were rotated on the agar pad to orient neuronal processes at the top plane (dorsoventral axis) before SDC microscopy. For dendrite, z-stack images covering the entire dendrite region were taken. For dorsal axon, z-stack images covering the presynaptic terminals (labelled by mCherry::RAB-3) were taken. The images were then projected by maximum projection. Background fluorescence was measured and subtracted from each maximum projected image, and entire dendrite and dorsal axon were selected for analysis. The clusters/ particles containing endoGFP::ESYT-2 signals above an arbitrary threshold were segmented, and area and mean fluorescent intensity of individual clusters/particles were measured using the "Analyze particles" command. The total fluorescence intensity was calculated as a sum of the product of the area and mean fluorescent intensity of individual clusters/particles and then plotted with normalization.

\section{Line scan analysis}

For analysis of the colocalization between endoGFP.::ESYT-2 and mCherry::RAB-3 puncta (synaptic vesicles) (Fig 2B) and between Reconstituted GFP (ER-PM contacts) and wrmScarlet::ESYT-2 (Fig $2 \mathrm{~F}$ ), line scan analysis was performed. A line of $50 \mu \mathrm{m}$ in length (Fig 2B) or $25 \mu \mathrm{m}$ in length (Fig 2F) was manually drawn along dorsal axons of DA9, and fluorescence intensity of GFP and mCherry/ wrmScarlet along the manually drawn line was measured. The value was normalized by the maximum fluorescence intensity and plotted.

For analysis of the localization of TRAP $\beta:: m N e o n G r e e n$ (Fig 6K), a line of $30 \mu \mathrm{m}$ in length was manually drawn along a dorsal axon of DA9, and fluorescence intensity of mNeonGreen along the manually drawn line was measured and plotted.

\section{Quantification of mNeonGreen::ESYT-2}

For quantification of the fluorescence intensity of mNeonGreen:: ESYT-2 along the different zones of DA9 neurons, z-stack images of the side view of a DA9 neuron were taken by SDC microscopy and projected into a single plane by maximum projection. Background fluorescence was measured in a region close to a DA9 neuron and subtracted from each maximum projected image. The backgroundsubtracted image was then converted to a 10-bit image, and the DA9 neuron was straightened using the "Straighten" function. A region covering the entire dendrite and a region of $70 \mu \mathrm{m}$ in width from the beginning of presynaptic terminals (marked by mCherry::RAB-3) was selected for analysis of dendrite and dorsal axon, respectively. The clusters/particles containing mNeonGreen::ESYT-2 signals above an arbitrary threshold were segmented, and area and mean fluorescent intensity of individual clusters/particles were measured using the "Analyze particles" command. The same threshold was used for all images. The total fluorescence intensity of mNeonGreen::ESYT-2 for different zones of a DA9 neuron was then calculated as a sum of the product of the area and mean fluorescent intensity of fluorescence clusters/particles (Figs $3 C-E-E, 5 B, S 4 C, S 5 D$ and $F$, and S6A). For some figures, the ratio of total fluorescence intensity of dorsal axon (or indicated regions of zone B for Fig S6F) to that of dendrite was plotted (Figs 3F, 4C and E, 5F, and 6C, S5G, S6F and G, and S7D). Occupancy of mNeonGreen:: ESYT-2 in dendrites was quantified by measuring the length of regions in dendrites that contained mNeonGreen::ESYT-2 (Figs 3G, $5 \mathrm{G}, \mathrm{S} 5 \mathrm{H}$, and $\mathrm{S} 6 \mathrm{~B})$.

\section{Quantification of mCherry::RAB-3}

For quantification of mCherry::RAB-3 puncta along the dorsal axon (Fig S3F), z-stack images of the side view a DA9 neuron were taken by SDC microscopy and projected into a single plane by maximum projection. Background fluorescence was measured in a region close to a DA9 neuron and subtracted from each maximum projected image. The background-subtracted image was then converted to a 10-bit image, and the DA9 neuron was straightened using the "Straighten" function. A region of dorsal axon covered by mCherry::RAB-3 puncta was selected for analysis. The clusters/ particles containing mCherry::RAB-3 signals above an arbitrary threshold were segmented, and the number of clusters/particles as well as total fluorescence intensity were measured using the "Analyze particles" command. 


\section{Quantification of cortical ER structure}

For quantification of ER structure (Figs 4G, 5I, 6A, and S6C), images of the periphery of DA9 neurons, expressing mNeonGreen::ESYT-2 (as a marker for cortical ER), were taken by SDC-SIM. The clusters/ particles containing mNeonGreen::ESYT-2 signals above an arbitrary threshold were segmented, and area of individual clusters/ particles were measured using the "Analyze particles" command. mNeonGreen::ESYT-2 fluorescence clusters that are less than 0.242 $\mu \mathrm{m}^{2}$ were considered as "tubules," and mNeonGreen::ESYT-2 fluorescence clusters that are more than $0.242 \mu \mathrm{m}^{2}$ were further analyzed based on their aspect ratio (AR) values; AR values more than 2.2 were considered as "tubules," whereas AR values less than 2.2 were considered as "sheets." The ratio of the area of cortical ER sheets to that of cortical ER tubules were then calculated and plotted.

\section{Quantification of ER length in dendrite}

For quantification of ER length in dendrites of DA9 neurons (Fig S5A), z-stack images of the side view a DA9 neuron were taken by SDC microscopy and projected into a single plane by maximum projection. ER length was quantified by measuring the length of regions in dendrites that contained CP450::mCherry.

\section{Quantification of rough ER marker}

For quantification of the fluorescence intensity of reconstituted GFP::SEC-61 (Figs $6 \mathrm{G}$ and $\mathrm{H}$ and S6H) or TRAP $\beta:: m$ NeonGreen (Figs 6) and S6I), z-stack images of the side view of a DA9 neuron were taken by SDC microscopy and were projected into a single plane by maximum projection. Background fluorescence was measured in a region close to a DA9 cell body and subtracted from each maximum projected image. After background subtraction, a region of $20 \mu \mathrm{m}$ in length was manually selected for dendrite (for both reconstituted GFP::SEC-61 $\beta$ and TRAP $\beta:: m$ NeonGreen) and dorsal axon (for reconstituted GFP::SEC-61 $\beta$ ). DA9 cell body was manually outlined for both reconstituted GFP::SEC-61 $\beta$ and TRAP $\beta:: m N e o n G r e e n$. The clusters/particles containing fluorescence signals above an arbitrary threshold were segmented, and area and mean fluorescence intensity of individual clusters/particles were measured using the "Analyze particles" command. The total fluorescence intensity was then calculated as sum of the product of the area and mean fluorescent intensity of fluorescence cluster/particles and plotted.

\section{Quantification of axon length after laser axotomy}

For quantification of axon length after laser axotomy (Fig 7D-G), z-stack images of the side view of a laser axotomized DA9 neuron were taken by SDC microscopy and were projected into a single plane by maximum projection. The full length of regenerated DA9 axons (labelled by SL2::mCherry) of recovered worms were measured from the beginning of dorsal axons. For some worms (e.g., wild-type control animals), multiple maximum projected images were used to measure the length of regenerated axons.

\section{Quantification of iRFP-PH ${ }^{P L C \delta 1}$ signals at the PM}

For analysis of the signal of iRFP-PH ${ }^{\mathrm{PLC} \delta 1}$ at the PM via SDC microscopy (Fig S4E and G), line scan analysis was performed. A line of $5 \mu \mathrm{m}$ in length was manually drawn around the PM, and iRFP fluorescence intensity along the manually drawn line was measured. The peak intensity around the PM region was normalized with the intensity of cytoplasmic region and then plotted for quantification.

\section{Statistical methods}

No statistical method was used to predetermine sample size, and the experiments were not randomized for live cell imaging. For comparing the mean of two groups, two-tailed unpaired $t$ test was performed. To compare the mean of multiple groups to the mean of an indicated group, one-way ANOVA followed by Dunnett's multiple comparisons test was performed. $P$-values $>0.05$ were considered not significant. " $\mathrm{n}$ " represents the number of animals shown in graphs. All data are shown as mean \pm SEM. In dot plots, each dot represents the value from a single worm (or a single cell) with the bar as the mean. At least eight animals (or cells) are scored in each experiment.

\section{Data Availability}

The authors declare that the data supporting the findings of this study are available within the paper and its supplementary information files. Source data for figures (Figs 1G, 2B, C, and F, 3C-G, 4C, E, G, and I, 5B, D, F, G, and I, 6A, C, E, G, H, J, and K, 7D-G, S1C, S3D-F, S4C, E, and G, S5A, D, and F-H, S6A-C and F-I, and S7B and D) are provided with the paper. Other data are available from the corresponding author upon reasonable request. Reagents and strains generated for this study are available directly from the authors upon request.

\section{Supplementary Information}

Supplementary Information is available at https://doi.org/10.26508/lsa. 202101092.

\section{Acknowledgements}

We thank Darshini Jeyasimman, Bilge Ercan, Dylan Hong Zheng Koh, and Dennis Dharmawan for sharing reagents and discussion. This work was supported in part by the Singapore Ministry of Education Academic Research Fund Tier 2 (MOE2017-T2-2-001), a Nanyang Assistant Professorship, a Lee Kong Chian School of Medicine startup grant (LKCMedicine-SUG), and an Ageing Research Institute for Society and Education (ARISE) seed grant (ARISE/2017/7) to Y Saheki.

\section{Author Contributions}

I Sun: data curation, formal analysis, validation, investigation, visualization, methodology, and writing-review and editing. R Harion: data curation, formal analysis, validation, investigation, visualization, methodology, and writing-review and editing. T Naito: data curation, formal analysis, validation, investigation, and visualization. 
Y Saheki: conceptualization, resources, data curation, supervision, funding acquisition, project administration, and writing-original draft, review, and editing.

\section{Conflict of Interest Statement}

The authors declare that they have no conflict of interest.

\section{References}

Anderson P (1995) Mutagenesis. Methods Cell Biol 48: 31-58. doi:10.1016/ s0091-679x(08)61382-5

Anwar K, Klemm RW, Condon A, Severin KN, Zhang M, Ghirlando R, Hu J, Rapoport TA, Prinz WA (2012) The dynamin-like GTPase Sey1p mediates homotypic ER fusion in S. cerevisiae. J Cell Biol 197: 209-217. doi:10.1083/jcb.201111115

Bae YK, Kim E, L'hernault SW, Barr MM (2009) The CIL-1 PI 5-phosphatase localizes TRP Polycystins to cilia and activates sperm in C. elegans. Curr Biol 19: 1599-1607. doi:10.1016/j.cub.2009.08.045

Bayer EM, Sparkes I, Vanneste S, Rosado A (2017) From shaping organelles to signalling platforms: The emerging functions of plant ER-PM contact sites. Curr Opin Plant Biol 40: 89-96. doi:10.1016/j.pbi.2017.08.006

Behrendt L, Kurth I, Kaether C (2019) A disease causing ATLASTIN 3 mutation affects multiple endoplasmic reticulum-related pathways. Cell Mol Life Sci 76: 1433-1445. doi:10.1007/s00018-019-03010-x

Besprozvannaya M, Dickson E, Li H, Ginburg KS, Bers DM, Auwerx J, Nunnari J (2018) GRAM domain proteins specialize functionally distinct ER-PM contact sites in human cells. Elife 7: e31019. doi:10.7554/eLife.31019

Bian X, Klemm RW, Liu TY, Zhang M, Sun S, Sui X, Liu X, Rapoport TA, Hu J (2011) Structures of the atlastin GTPase provide insight into homotypic fusion of endoplasmic reticulum membranes. Proc Natl Acad Sci U S A 108: 3976-3981. doi:10.1073/pnas. 1101643108

Bian X, Saheki Y, De Camilli P (2018) Ca2+ releases E-Syt1 autoinhibition to couple ER-plasma membrane tethering with lipid transport. EMBO J 37: 219-234. doi:10.15252/embj.201797359

Blackstone C (2012) Cellular pathways of hereditary spastic paraplegia. Annu Rev Neurosci 35: 25-47. doi:10.1146/annurev-neuro-062111-150400

Brenner S (1974) The genetics of Caenorhabditis elegans. Genetics 77: 71-94. doi:10.1093/genetics/77.1.71

Byrnes LJ, Sondermann H (2011) Structural basis for the nucleotidedependent dimerization of the large $\mathrm{G}$ protein atlastin-1/SPG3A. Proc Natl Acad Sci U S A 108: 2216-2221. doi:10.1073/pnas.1012792108

Chang CL, Hsieh TS, Yang TT, Rothberg KG, Azizoglu DB, Volk E, Liao JC, Liou J (2013) Feedback regulation of receptor-induced Ca2+ signaling mediated by E-Syt1 and Nir2 at endoplasmic reticulum-plasma membrane junctions. Cell Rep 5: 813-825. doi:10.1016/ j.celrep.2013.09.038

Chen YJ, Quintanilla CG, Liou J (2019) Recent insights into mammalian ER-PM junctions. Curr Opin Cell Biol 57: 99-105. doi:10.1016/j.ceb.2018.12.011

Cieri D, Vicario M, Giacomello M, Vallese F, Filadi R, Wagner T, Pozzan T, Pizzo P, Scorrano L, Brini M, et al (2018) SPLICS: A split green fluorescent protein-based contact site sensor for narrow and wide heterotypic organelle juxtaposition. Cell Death Differ 25: 1131-1145. doi:10.1038/ s41418-017-0033-z

Collado J, Kalemanov M, Campelo F, Bourgoint C, Thomas F, Loewith R, Martínez-Sánchez A, Baumeister W, Stefan CJ, Fernández-Busnadiego R (2019) Tricalbin-mediated contact sites control ER curvature to maintain plasma membrane integrity. Dev Cell 51: 476-487.e7. doi:10.1016/j.devcel.2019.10.018
Cui-Wang T, Hanus C, Cui T, Helton T, Bourne J, Watson D, Harris KM, Ehlers MD (2012) Local zones of endoplasmic reticulum complexity confine cargo in neuronal dendrites. Cell 148: 309-321. doi:10.1016/j.cell.2011.11.056

De Craene JO, Coleman J, Estrada de Martin P, Pypaert M, Anderson S, Yates JR 3rd, Ferro-Novick S, Novick P (2006) Rtn1p is involved in structuring the cortical endoplasmic reticulum. Mol Biol Cell 17: 3009-3020. doi:10.1091/mbc.e06-01-0080

de Juan-Sanz J, Holt GT, Schreiter ER, de Juan F, Kim DS, Ryan TA (2017) Axonal endoplasmic reticulum $\mathrm{Ca} 2+$ content controls release probability in CNS nerve terminals. Neuron 93: 867-881.e6. doi:10.1016/ j.neuron.2017.01.010

Dejima K, Hori S, Iwata S, Suehiro Y, Yoshina S, Motohashi T, Mitani S (2018) An aneuploidy-free and structurally defined balancer chromosome toolkit for Caenorhabditis elegans. Cell Rep 22: 232-241. doi:10.1016/ j.celrep.2017.12.024

Ding C, Hammarlund M (2018) Aberrant information transfer interferes with functional axon regeneration. Elife 7: e38829. doi:10.7554/eLife.38829

Dong R, Zhu T, Benedetti L, Gowrishankar S, Deng H, Cai Y, Wang X, Shen K, De Camilli P (2018) The inositol 5-phosphatase INPP5K participates in the fine control of ER organization. J Cell Biol 217: 3577-3592. doi:10.1083/ jcb.201802125

El Mouridi S, Lecroisey C, Tardy P, Mercier M, Leclercq-Blondel A, Zariohi N, Boulin T (2017) Reliable CRISPR/Cas9 genome engineering in Caenorhabditis elegans using a single efficient sgRNA and an easily recognizable phenotype. G3 (Bethesda) 7: 1429-1437. doi:10.1534/g3.117.040824

Espadas J, Pendin D, Bocanegra R, Escalada A, Misticoni G, Trevisan T, Velasco Del Olmo A, Montagna A, Bova S, Ibarra B, et al (2019) Dynamic constriction and fission of endoplasmic reticulum membranes by reticulon. Nat Commun 10: 5327. doi:10.1038/s41467-019-13327-7

Farías GG, Fréal A, Tortosa E, Stucchi R, Pan X, Portegies S, Will L, Altelaar M, Hoogenraad CC (2019) Feedback-driven mechanisms between microtubules and the endoplasmic reticulum instruct neuronal polarity. Neuron 102: 184-201.e8. doi:10.1016/j.neuron.2019.01.030

Fernandez-Busnadiego R, Saheki Y, De Camilli P (2015) Three-dimensional architecture of extended synaptotagmin-mediated endoplasmic reticulum-plasma membrane contact sites. Proc Natl Acad Sci U S A 112: E2004-E2013. doi:10.1073/pnas.1503191112

Fink KL, López-Giráldez F, Kim IJ, Strittmatter SM, Cafferty WBJ (2017) Identification of intrinsic axon growth modulators for intact CNS neurons after injury. Cell Rep 18: 2687-2701. doi:10.1016/ j.celrep.2017.02.058

Fischer D, Schabhüttl M, Wieland T, Windhager R, Strom TM, Auer-Grumbach M (2014) A novel missense mutation confirms ATL3 as a gene for hereditary sensory neuropathy type 1. Brain 137: e286. doi:10.1093/ brain/awu091

Friedman JR, Voeltz GK (2011) The ER in 3D: A multifunctional dynamic membrane network. Trends Cell Biol 21: 709-717. doi:10.1016/ j.tcb.2011.07.004

Giordano F, Saheki Y, Idevall-Hagren O, Colombo SF, Pirruccello M, Milosevic I, Gracheva EO, Bagriantsev SN, Borgese N, De Camilli P (2013) PI(4,5) $\mathrm{P}(2)$-dependent and $\mathrm{Ca}(2+)$-regulated ER-PM interactions mediated by the extended synaptotagmins. Cell 153: 1494-1509. doi:10.1016/ j.cell.2013.05.026

Gurung R, Tan A, Ooms LM, McGrath MJ, Huysmans RD, Munday AD, Prescott M, Whisstock JC, Mitchell CA (2003) Identification of a novel domain in two mammalian inositol-polyphosphate 5-phosphatases that mediates membrane ruffle localization. The inositol 5-phosphatase skip localizes to the endoplasmic reticulum and translocates to membrane ruffles following epidermal growth factor stimulation. I Biol Chem 278: 11376-11385. doi:10.1074/jbc.M209991200

Hammarlund M, Nix P, Hauth L, Jorgensen EM, Bastiani M (2009) Axon regeneration requires a conserved MAP kinase pathway. Science 323: 802-806. doi:10.1126/science.1165527 
Hoffmann PC, Bharat TAM, Wozny MR, Boulanger J, Miller EA, Kukulski W (2019) Tricalbins contribute to cellular lipid flux and form curved ER-PM contacts that are bridged by rod-shaped structures. Dev Cell 51: 488-502.e8. doi:10.1016/j.devcel.2019.09.019

Hu J, Shibata Y, Voss C, Shemesh T, Li Z, Coughlin M, Kozlov MM, Rapoport TA, Prinz WA (2008) Membrane proteins of the endoplasmic reticulum induce high-curvature tubules. Science 319: 1247-1250. doi:10.1126/science.1153634

Hu J, Shibata Y, Zhu PP, Voss C, Rismanchi N, Prinz WA, Rapoport TA, Blackstone C (2009) A class of dynamin-like GTPases involved in the generation of the tubular ER network. Cell 138: 549-561. doi:10.1016/ j.cell.2009.05.025

Idevall-Hagren O, Lü A, Xie B, De Camilli P (2015) Triggered Ca2+ influx is required for extended synaptotagmin 1-induced ER-plasma membrane tethering. EMBO / 34: 2291-2305. doi:10.15252/embj.201591565

Ijuin T, Mochizuki Y, Fukami K, Funaki M, Asano T, Takenawa T (2000) Identification and characterization of a novel inositol polyphosphate 5-phosphatase. J Biol Chem 275: 10870-10875. doi:10.1074/ jbc.275.15.10870

Ijuin T, Yu YE, Mizutani K, Pao A, Tateya S, Tamori Y, Bradley A, Takenawa T (2008) Increased insulin action in SKIP heterozygous knockout mice. Mol Cell Biol 28: 5184-5195. doi:10.1128/MCB.01990-06

Jeyasimman D, Saheki Y (2019) SMP domain proteins in membrane lipid dynamics. Biochim Biophys Acta Mol Cell Biol Lipids 1865: 158447. doi:10.1016/j.bbalip.2019.04.007

Johnson B, Leek AN, Solé L, Maverick EE, Levine TP, Tamkun MM (2018) Kv2 potassium channels form endoplasmic reticulum/plasma membrane junctions via interaction with VAPA and VAPB. Proc Natl Acad Sci U S A 115: E7331-E7340. doi:10.1073/pnas.1805757115

Kakimoto Y, Tashiro S, Kojima R, Morozumi Y, Endo T, Tamura Y (2018) Visualizing multiple inter-organelle contact sites using the organelletargeted split-GFP system. Sci Rep 8: 6175. doi:10.1038/s41598-018-24466-0

Kakizawa S, Kishimoto Y, Hashimoto K, Miyazaki T, Furutani K, Shimizu H, Fukaya M, Nishi M, Sakagami H, Ikeda A, et al (2007) Junctophilinmediated channel crosstalk essential for cerebellar synaptic plasticity. EMBO / 26: 1924-1933. doi:10.1038/sj.emboj.7601639

Kamiyama D, Sekine S, Barsi-Rhyne B, Hu J, Chen B, Gilbert LA, Ishikawa H, Leonetti MD, Marshall WF, Weissman JS, et al (2016) Versatile protein tagging in cells with split fluorescent protein. Nat Commun 7: 11046. doi:10.1038/ncomms11046

Kim KW, Tang NH, Piggott CA, Andrusiak MG, Park S, Zhu M, Kurup N, Cherra S) 3rd, Wu Z, Chisholm AD, et al (2018) Expanded genetic screening in Caenorhabditis elegans identifies new regulators and an inhibitory role for NAD+ in axon regeneration. Elife 7: e39756. doi:10.7554/eLife.39756

Kirmiz M, Gillies TE, Dickson EJ, Trimmer JS (2019) Neuronal ER-plasma membrane junctions organized by KV2-VAP pairing recruit Nir proteins and affect phosphoinositide homeostasis. I Biol Chem 294: 17735-17757. doi:10.1074/jbc.RA119.007635

Klassen MP, Shen K (2007) Wnt signaling positions neuromuscular connectivity by inhibiting synapse formation in C. elegans. Cell 130: 704-716. doi:10.1016/j.cell.2007.06.046

Kornak U, Mademan I, Schinke M, Voigt M, Krawitz P, Hecht J, Barvencik F, Schinke T, Giesselmann S, Beil FT, et al (2014) Sensory neuropathy with bone destruction due to a mutation in the membrane-shaping atlastin GTPase 3. Brain 137: 683-692. doi:10.1093/brain/awt357

Krols M, Asselbergh B, De Rycke R, De Winter V, Seyer A, Müller FJ, Kurth I, Bultynck G, Timmerman V, Janssens S (2019) Sensory neuropathycausing mutations in ATL3 affect ER-mitochondria contact sites and impair axonal mitochondrial distribution. Hum Mol Genet 28: 615-627. doi:10.1093/hmg/ddy352

Krols M, Detry S, Asselbergh B, Almeida-Souza L, Kremer A, Lippens S, De Rycke R, De Winter V, Müller FJ, Kurth I, et al (2018) Sensory-neuropathycausing mutations in ATL3 cause aberrant ER membrane tethering. Cell Rep 23: 2026-2038. doi:10.1016/j.celrep.2018.04.071
Liu X, Guo X, Niu L, Li X, Sun F, Hu J, Wang X, Shen K (2019) Atlastin-1 regulates morphology and function of endoplasmic reticulum in dendrites. Nat Commun 10: 568. doi:10.1038/s41467-019-08478-6

Manford AG, Stefan CJ, Yuan HL, Macgurn JA, Emr SD (2012) ER-to-plasma membrane tethering proteins regulate cell signaling and $E R$ morphology. Dev Cell 23: 1129-1140. doi:10.1016/j.devcel.2012.11.004

Mello C, Fire A (1995) DNA transformation. Methods Cell Biol 48: 451-482. doi:10.1016/s0091-679x(08)61399-0

Moriguchi S, Nishi M, Komazaki S, Sakagami H, Miyazaki T, Masumiya H, Saito $\mathrm{SY}$, Watanabe $\mathrm{M}$, Kondo $\mathrm{H}$, Yawo $\mathrm{H}$, et al (2006) Functional uncoupling between $\mathrm{Ca} 2+$ release and afterhyperpolarization in mutant hippocampal neurons lacking junctophilins. Proc Natl Acad Sci U S A 103: 10811-10816. doi:10.1073/pnas.0509863103

Nath VR, Mishra S, Basak B, Trivedi D, Raghu P (2020) Extended synaptotagmin regulates membrane contact site structure and lipid transfer function in vivo. EMBO Rep 21: e50264. doi:10.15252/embr.202050264

O'Sullivan NC, Jahn TR, Reid E, O'Kane CJ (2012) Reticulon-like-1, the Drosophila orthologue of the hereditary spastic paraplegia gene reticulon 2 , is required for organization of endoplasmic reticulum and of distal motor axons. Hum Mol Genet 21: 3356-3365. doi:10.1093/hmg/dds167

Orci L, Ravazzola M, Le Coadic M, Shen WW, Demaurex N, Cosson P (2009) From the cover: STIM1-induced precortical and cortical subdomains of the endoplasmic reticulum. Proc Natl Acad Sci U S A 106: 19358-19362. doi:10.1073/pnas.0911280106

Orso G, Pendin D, Liu S, Tosetto J, Moss TJ, Faust JE, Micaroni M, Egorova A, Martinuzzi A, McNew JA, et al (2009) Homotypic fusion of ER membranes requires the dynamin-like GTPase atlastin. Nature 460: 978-983. doi:10.1038/nature08280

Osborn DPS, Pond HL, Mazaheri N, Dejardin J, Munn CJ, Mushref K, Cauley ES, Moroni I, Pasanisi MB, Sellars EA, et al (2017) Mutations in INPP5K cause a form of congenital muscular dystrophy overlapping marinesco-sjögren syndrome and dystroglycanopathy. Am J Hum Genet 100: 537-545. doi:10.1016/j.ajhg.2017.01.019

Öztürk Z, O'Kane CJ, Pérez-Moreno JJ (2020) Axonal endoplasmic reticulum dynamics and its roles in neurodegeneration. Front Neurosci 14: 48. doi:10.3389/fnins.2020.00048

Petkovic M, Jemaiel A, Daste F, Specht CG, Izeddin I, Vorkel D, Verbavatz JM, Darzacq X, Triller A, Pfenninger KH, et al (2014) The SNARE Sec22b has a non-fusogenic function in plasma membrane expansion. Nat Cell Biol 16: 434-444. doi:10.1038/ncb2937

Phillips MJ, Voeltz GK (2016) Structure and function of ER membrane contact sites with other organelles. Nat Rev Mol Cell Biol 17: 69-82. doi:10.1038/nrm.2015.8

Piggott CA, Wu Z, Nurrish S, Xu S, Kaplan JM, Chisholm AD, Jin Y (2021) Caenorhabditis elegans Junctophilin has tissue-specific functions and regulates neurotransmission with extended-synaptotagmin. Genetics 218: iyab063. doi:10.1093/genetics/iyab063

Pokala N, Liu Q, Gordus A, Bargmann Cl (2014) Inducible and titratable silencing of Caenorhabditis elegans neurons in vivo with histaminegated chloride channels. Proc Natl Acad Sci U S A 111: 2770-2775. doi:10.1073/pnas.1400615111

Ramírez OA, Couve A (2011) The endoplasmic reticulum and protein trafficking in dendrites and axons. Trends Cell Biol 21: 219-227. doi:10.1016/j.tcb.2010.12.003

Rolls MM, Hall DH, Victor M, Stelzer EH, Rapoport TA (2002) Targeting of rough endoplasmic reticulum membrane proteins and ribosomes in invertebrate neurons. Mol Biol Cell 13: 1778-1791. doi:10.1091/mbc.01-10-0514

Romei MG, Boxer SG (2019) Split green fluorescent proteins: Scope, limitations, and outlook. Annu Rev Biophys 48: 19-44. doi:10.1146/ annurev-biophys-051013-022846

Roth S, Heintzmann R (2016) Optical photon reassignment with increased axial resolution by structured illumination. Methods Appl Fluoresc 4: 045005. doi:10.1088/2050-6120/4/4/045005 
Saheki Y (2017) Endoplasmic reticulum-plasma membrane crosstalk mediated by the extended synaptotagmins. Adv Exp Med Biol 997: 83-93. doi:10.1007/978-981-10-4567-7_6

Saheki Y, Bargmann CI (2009) Presynaptic CaV2 calcium channel traffic requires CALF-1 and the alpha(2)delta subunit UNC-36. Nat Neurosci 12: 1257-1265. doi:10.1038/nn.2383

Saheki Y, Bian X, Schauder CM, Sawaki Y, Surma MA, Klose C, Pincet F, Reinisch KM, De Camilli P (2016) Control of plasma membrane lipid homeostasis by the extended synaptotagmins. Nat Cell Biol 18: 504-515. doi:10.1038/ncb3339

Saheki Y, De Camilli P (2017a) Endoplasmic reticulum-plasma membrane contact sites. Annu Rev Biochem 86: 659-684. doi:10.1146/annurevbiochem-061516-044932

Saheki Y, De Camilli P (2017b) The extended-synaptotagmins. Biochim Biophys Acta Mol Cell Res 1864: 1490-1493. doi:10.1016/ j.bbamcr.2017.03.013

Schauder CM, Wu X, Saheki Y, Narayanaswamy P, Torta F, Wenk MR, De Camilli $P$, Reinisch KM (2014) Structure of a lipid-bound extended synaptotagmin indicates a role in lipid transfer. Nature 510: 552-555. doi:10.1038/nature13269

Schmid AC, Wise HM, Mitchell CA, Nussbaum R, Woscholski R (2004) Type II phosphoinositide 5-phosphatases have unique sensitivities towards fatty acid composition and head group phosphorylation. FEBS Lett 576: 9-13. doi:10.1016/j.febslet.2004.08.052

Schwartz ML, Jorgensen EM (2016) SapTrap, a toolkit for high-throughput CRISPR/Cas9 gene modification in Caenorhabditis elegans. Genetics 202: 1277-1288. doi:10.1534/genetics.115.184275

Schwarz DS, Blower MD (2016) The endoplasmic reticulum: Structure, function and response to cellular signaling. Cell Mol Life Sci 73: 79-94. doi:10.1007/s00018-015-2052-6

Sclip A, Bacaj T, Giam LR, Südhof TC (2016) Extended synaptotagmin (ESyt) triple knock-out mice are viable and fertile without obvious endoplasmic reticulum dysfunction. PLoS One 11: e0158295. doi:10.1371/journal.pone.0158295

Shai N, Yifrach E, van Roermund CWT, Cohen N, Bibi C, IJlst L, Cavellini L, Meurisse J, Schuster R, Zada L, et al (2018) Systematic mapping of contact sites reveals tethers and a function for the peroxisome-mitochondria contact. Nat Commun 9: 1761. doi:10.1038/s41467-018-03957-8

Shibata Y, Hu J, Kozlov MM, Rapoport TA (2009) Mechanisms shaping the membranes of cellular organelles. Annu Rev Cell Dev Biol 25: 329-354. doi:10.1146/annurev.cellbio.042308.113324

Shibata Y, Shemesh T, Prinz WA, Palazzo AF, Kozlov MM, Rapoport TA (2010) Mechanisms determining the morphology of the peripheral ER. Cell 143: 774-788. doi:10.1016/j.cell.2010.11.007

Shibata Y, Voeltz GK, Rapoport TA (2006) Rough sheets and smooth tubules. Cell 126: 435-439. doi:10.1016/j.cell.2006.07.019

Stefan CJ (2020) Endoplasmic reticulum-plasma membrane contacts: Principals of phosphoinositide and calcium signaling. Curr Opin Cell Biol 63: 125-134. doi:10.1016/j.ceb.2020.01.010

Summerville JB, Faust JF, Fan E, Pendin D, Daga A, Formella J, Stern M, McNew JA (2016) The effects of ER morphology on synaptic structure and function in Drosophila melanogaster. J Cell Sci 129: 1635-1648. doi:10.1242/jcs.184929

Sun EW, Guillén-Samander A, Bian X, Wu Y, Cai Y, Messa M, De Camilli P (2019) Lipid transporter TMEM24/C2CD2L is a Ca2+-regulated component of ER-plasma membrane contacts in mammalian neurons. Proc Natl Acad Sci U S A 116: 5775-5784. doi:10.1073/pnas.1820156116

Terasaki M (2018) Axonal endoplasmic reticulum is very narrow. J Cell Sci 131: jcs210450. doi:10.1242/jcs.210450

Terasaki M, Shemesh T, Kasthuri N, Klemm RW, Schalek R, Hayworth KJ, Hand AR, Yankova M, Huber G, Lichtman JW, et al (2013) Stacked endoplasmic reticulum sheets are connected by helicoidal membrane motifs. Cell 154: 285-296. doi:10.1016/j.cell.2013.06.031

Terasaki M, Slater NT, Fein A, Schmidek A, Reese TS (1994) Continuous network of endoplasmic reticulum in cerebellar Purkinje neurons Proc Natl Acad Sci U S A 91: 7510-7514. doi:10.1073/pnas.91.16.7510

Toulmay A, Prinz WA (2012) A conserved membrane-binding domain targets proteins to organelle contact sites. J Cell Sci 125: 49-58. doi:10.1242/jcs.085118

Tremblay MG, Moss T (2016) Loss of all 3 Extended Synaptotagmins does not affect normal mouse development, viability or fertility. Cell Cycle 15 2360-2366. doi:10.1080/15384101.2016.1203494

Tsukita S, Ishikawa H (1976) Three-dimensional distribution of smooth endoplasmic reticulum in myelinated axons. J Electron Microsc (Tokyo) 25: 141-149. doi:10.1093/oxfordjournals.jmicro.a050013

Valm AM, Cohen S, Legant WR, Melunis J, Hershberg U, Wait E, Cohen AR, Davidson MW, Betzig E, Lippincott-Schwartz J (2017) Applying systemslevel spectral imaging and analysis to reveal the organelle interactome. Nature 546: 162-167. doi:10.1038/ nature22369

Vierra NC, Kirmiz M, van der List D, Santana LF, Trimmer JS (2019) Kv2.1 mediates spatial and functional coupling of L-type calcium channels and ryanodine receptors in mammalian neurons. Elife 8: e49953. doi:10.7554/eLife.49953

Voeltz GK, Prinz WA, Shibata Y, Rist JM, Rapoport TA (2006) A class of membrane proteins shaping the tubular endoplasmic reticulum. Cell 124: 573-586. doi:10.1016/j.cell.2005.11.047

Wang S, Tukachinsky H, Romano FB, Rapoport TA (2016) Cooperation of the ER-shaping proteins atlastin, lunapark, and reticulons to generate a tubular membrane network. Elife 5: e18605. doi:10.7554/eLife.18605

West M, Zurek N, Hoenger A, Voeltz GK (2011) A 3D analysis of yeast ER structure reveals how ER domains are organized by membrane curvature. J Cell Biol 193: 333-346. doi:10.1083/jcb.201011039

Westrate LM, Lee JE, Prinz WA, Voeltz GK (2015) Form follows function: The importance of endoplasmic reticulum shape. Annu Rev Biochem 84: 791-811. doi:10.1146/annurev-biochem-072711-163501

Wiessner M, Roos A, Munn CJ, Viswanathan R, Whyte T, Cox D, Schoser B, Sewry C, Roper H, Phadke R, et al (2017) Mutations in INPP5K, encoding a phosphoinositide 5-phosphatase, cause congenital muscular dystrophy with cataracts and mild cognitive impairment. Am J Hum Genet 100: 523-536. doi:10.1016/ j.ajhg.2017.01.024

Wu H, Carvalho P, Voeltz GK (2018) Here, there, and everywhere: The importance of ER membrane contact sites. Science 361: eaan5835. doi:10.1126/science.aan5835

Wu Y, Whiteus C, Xu CS, Hayworth KJ, Weinberg RJ, Hess HF, De Camilli P (2017) Contacts between the endoplasmic reticulum and other membranes in neurons. Proc Natl Acad Sci U S A 114: E4859-E4867. doi:10.1073/ pnas. 1701078114

Yalçın B, Zhao L, Stofanko M, O'Sullivan NC, Kang ZH, Roost A, Thomas MR, Zaessinger S, Blard O, Patto AL, et al (2017) Modeling of axonal endoplasmic reticulum network by spastic paraplegia proteins. Elife 6: e23882. doi:10.7554/eLife.23882

York AG, Parekh SH, Dalle Nogare D, Fischer RS, Temprine K, Mione M, Chitnis AB, Combs CA, Shroff H (2012) Resolution doubling in live, multicellular organisms via multifocal structured illumination microscopy. Nat Methods 9: 749-754. doi:10.1038/nmeth.2025

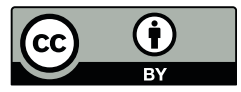

License: This article is available under a Creative Commons License (Attribution 4.0 International, as described at https://creativecommons.org/ licenses/by/4.0/). 